\title{
The Contribution of Color to Motion Processing in Macaque Middle Temporal Area
}

\author{
Alexander Thiele, ${ }^{1}$ Karen R. Dobkins, ${ }^{2}$ and Thomas D. Albright ${ }^{1,3}$ \\ 1 The Salk Institute for Biological Studies, San Diego, California 92186, ${ }^{2}$ Department of Psychology, University of California \\ San Diego, La Jolla, California 92093-0109, and 3Howard Hughes Medical Institute, La Jolla, California 92093-0646
}

The chromatic properties of an image yield strong cues for object boundaries and thus hold the potential to facilitate the detection of object motion. The extent to which cortical motion detectors exploit chromatic information, however, remains a matter of debate. To address this further, we quantified the strength of chromatic input to directionally selective neurons in the middle temporal area (MT) of macaque cerebral cortex using an equivalent luminance contrast (EqLC) paradigm. This paradigm, in which two sinusoidal gratings, one heterochromatic and the other achromatic, are superimposed and moved in opposite directions, allows the sensitivity of motion detectors to heterochromatic stimuli to be quantified and expressed relative to the benchmark of sensitivity for a luminance-defined stimulus.

The results of these experiments demonstrate that the chromatic contrast in a moving red-green heterochromatic grating strongly influences directional responses in MT when the lumi-

The extent to which the primate motion system uses information about the chromatic properties of an image is an issue of longstanding debate. Early experiments suggested a channeling of image information through several stages of the visual hierarchy, such that color is encoded predominantly within parvocellular and ventral cortical visual areas, whereas motion is processed primarily by neurons of the magnocellular and dorsal cortical stream (for review, see Merigan and Maunsell, 1993; Dobkins and Albright, 1998). More recent anatomical, physiological, and psychophysical evidence, however, indicates that this functional dichotomy is less complete (Schiller and Malpelli, 1978; Cavanagh et al., 1984; Derrington and Badcock, 1985; Mullen and Baker, 1985; Lindsey and Teller, 1990; Maunsell et al., 1990; Cavanagh and Anstis, 1991; Ferrera et al., 1992, 1994a; Dobkins and Albright, 1993; Nealey and Maunsell, 1994; Yoshioka et al., 1994; Gegenfurtner and Hawken, 1995; Sawatari et al., 1996; Croner and Albright, 1997).

Efforts to identify the substrate and mechanisms responsible for the use of chromatic information by motion detectors have focused on the middle temporal area (MT) of macaques. Contrary to the selective channeling hypothesis, many MT neurons exhibit directional selectivity when presented with isoluminant stimuli, although selectivity is diminished relative to that for

Received Jan. 19, 1999; revised April 29, 1999; accepted May 6, 1999.

We like to thank J. Constanza and K. Sevenbergen for technical assistance. A.T. was supported by the Human Frontier Science Program. T.D.A. is an investigator of the Howard Hughes Medical Institute.

Correspondence should be addressed to Alexander Thiele, The Salk Institute for Biological Studies, P.O. Box 85800, San Diego, CA 92186.

Copyright (C) 1999 Society for Neuroscience $0270-6474 / 99 / 196571-17 \$ 05.00 / 0$ nance contrast in that same grating is relatively low; for such stimuli, EqLC is at least $5 \%$. When luminance contrast is added to the heterochromatic grating, however, EqLC wanes sharply and becomes negative $(-4 \%)$ when luminance contrast is sufficiently high (>17-23\%). Thus, the chromatic properties of an object appear to confer little or no benefit to motion processing by MT neurons when sufficient luminance contrast concurrently exists. These data support a simple model in which chromatic motion processing in MT is almost exclusively determined by magnocellular input. Additionally, a comparison of neuronal and psychophysical data suggests that MT may not be the sole contributor to the perceptual experience elicited by motion of heterochromatic patterns, or that only a subset of MT neurons serve this function.

Key words: luminance; chrominance; magnocellular; parvocellular; contrast; extrastriate

luminance-defined stimuli (Charles and Logothetis, 1989; Saito et al., 1989; Dobkins and Albright, 1990, 1994, 1998; Gegenfurtner et al., 1994).

Based on our previous results, we have argued that the observed chromatic influences in MT can be accounted for by signals originating in magnocellular (M) subcortical areas (Dobkins and Albright, 1994, 1998). Although M-cells are generally thought to possess broad-band spectral sensitivity, there are two ways by which they convey information about chromatic contrast. First, individual M-cells signal the presence of chromatic contrast in isoluminant patterns without regard for chromatic contrast polarity (Schiller and Colby, 1983; Derrington et al., 1984; Lee et al., 1988, 1989a,b; Kaiser et al., 1990; Logothetis et al., 1990; Valberg et al., 1992; Dacey, 1996). Second, because isoluminant points vary across the population of M-cells, some cells will transmit a signal for any heterochromatic luminance ratio selected (Logothetis et al., 1990).

Human psychophysical results, by contrast, are more indicative of chromatic input to motion detectors arising in the parvocellular (P) stream (Cavanagh and Anstis, 1991; Gegenfurtner and Hawken, 1995; Stromeyer et al., 1995; Cropper and Derrington, 1996). In particular, Cavanagh and Anstis (1991) used a paradigm that allowed the sensitivity of motion detectors to heterochromatic stimuli to be quantified relative to sensitivity to a luminance-defined standard. The resulting metric was termed "equivalent luminance contrast" (EqLC). Cavanagh and Anstis (1991) reported that EqLC for red-green gratings was $\sim 10 \%$, which was viewed as a rather potent contribution. Moreover, sensitivity to chromatic contrast (as measured by EqLC) was 
independent of the luminance contrast in the moving stimulus, as would be expected from chromatically opponent (P-cell) input to motion detectors.

There are a number of possible explanations for the discrepant interpretations, M-cell versus P-cell hypotheses, derived from the aforementioned data. Among those worthy of consideration are (1) the different results have arisen because physiological and psychophysical data were obtained under different stimulus conditions; and (2) MT may not be the primary neuronal substrate for perception of chromatically defined motion, as suggested by Gegenfurtner et al. (1994). The present experiments were designed to address the first possibility directly (and thereby indirectly explore the legitimacy of the second) through use of a common design for neurophysiological and psychophysical experiments. Specifically, both neuronal and perceptual measures of EqLC for moving heterochromatic stimuli were obtained. These estimates (1) precisely quantify chromatic sensitivity relative to a luminance benchmark, (2) identify the relative contributions of coexistent chromatic and luminance contrast (a common realworld occurrence), and (3) bear on the relative validity of M- and P-cell hypotheses.

Our physiological findings demonstrate that the average sensitivity of MT neurons to a heterochromatic stimulus depends heavily on the degree of luminance contrast in the stimulus. This pattern of results can be accounted for by a model that assumes M-cell input. These physiological findings are in sharp contrast to our psychophysical confirmation of a nearly independent relationship between EqLC and luminance contrast. Confronted with this discrepancy, we consider the possibility that MT may not be the sole contributor to the perceptual experience elicited by motion of heterochromatic patterns or that only a small subset of MT neurons, those that match human psychophysical performance, serve this function.

\section{MATERIALS AND METHODS}

\section{General}

The results reported herein were obtained from behavioral and electrophysiological experiments in monkeys and psychophysical experiments conducted on human subjects. The stimulus configuration, display characteristics, viewing parameters, and behavioral requirements were identical for monkeys and humans except where noted below.

\section{Animal preparation and training}

Animal subjects. Two adult rhesus monkeys (Macaca mulatta, one male and one female) were used in this study. Experimental protocols were approved by the Salk Institute Animal Care and Use Committee, and conform to US Department of Agriculture regulations and to the National Institutes of Health guidelines for the humane care and use of laboratory animals.

Surgical preparation. Procedures for surgery and wound maintenance have been described in detail elsewhere (Dobkins and Albright, 1994). Briefly, a head post and a recording cylinder were affixed to the skull using stainless steel rails and screws (Synthes) and dental acrylic. Cranial magnetic resonance imaging (MRI) scans performed before surgery aided positioning of the recording chamber above MT (chambers were centered $17 \mathrm{~mm}$ lateral and $4 \mathrm{~mm}$ posterior to the midsagittal and interaural plane). A search coil for measuring eye position was surgically implanted in one eye using a variation of the method of Judge et al. (1980). The wire leads were connected to a two-pin miniconnector affixed to the cranial implant using dental acrylic. After surgical recovery and attainment of criterion performance on a visual fixation task (see below), a craniotomy was performed to allow for electrode passage into area MT. All surgical procedures were performed under sterile conditions, and animals were given prophylactic antibiotics (three doses of 30 $\mathrm{mg} / \mathrm{kg}$ Keflin during surgery at $2 \mathrm{hr}$ intervals) and postsurgical analgesics (buprenophorine, $0.03 \mathrm{mg} / \mathrm{kg}$, i.m., every $12 \mathrm{hr}$ for $3 \mathrm{~d}$ ).

Behavioral task. Monkeys were seated in a standard primate chair
(Christ Instruments) with head post rigidly supported by the frame of the chair. Animals were required to fixate a small $\left(0.2^{\circ}\right.$ diameter $)$ fixation target in the presence of moving visual stimuli for the duration of each trial $(1700 \mathrm{msec})$. The target was presented on a video display at a viewing distance of $60 \mathrm{~cm}$. Eye position was monitored (sampling rate, 60 $\mathrm{Hz}$ ) using the magnetic scleral search coil technique (Robinson, 1963). After successful fixation (i.e., the maintenance of eye position within a $2^{\circ}$ window centered on the fixation target) throughout the trial, animals were given a small $(\sim 0.15 \mathrm{cc})$ juice reward. When the animal broke fixation, the trial was terminated, and a one second "time-out" ensued.

\section{Electrophysiological recordings}

Our procedures for recording extracellular action potentials from isolated cortical neurons are routine and have been described in detail elsewhere (Dobkins and Albright, 1994). The following criteria were used to establish that recordings were from area MT: (1) a large proportion of highly direction-selective cells, (2) retinotopic organization consistent with known topography, (3) receptive field sizes consistent with known dependence on visual field eccentricity, and (4) electrode position relative to sulcal topography. In addition, stereotaxic MRI scans obtained before surgery were used to further confirm that our recordings were in a region of cortex consistent with the typical location of area MT.

\section{Apparatus}

Visual stimuli were generated using a SGT Pepper graphics board [Number Nine Computer; $640 \times 480$ pixel resolution, analog red-greenblue (RGB) output, 8 bits per gun) residing in a Pentium-based personal computer. Stimuli were displayed on a 20 inch analog RGB monitor (Sony GDM 2000TC; $60 \mathrm{~Hz}$, noninterlaced). Stimuli were generated under the charge of CORTEX 5.7 (Laboratory of Neuropsychology, National Institute of Mental Health), which was also used for data acquisition and behavioral control. Linearization of monitor output was achieved for each of the three phosphors independently (Watson et al., 1986).

\section{Visual stimuli}

General features. These experiments used moving sinusoidal gratings for visual stimulation. The mean luminance of all stimuli was $24 \mathrm{~cd} / \mathrm{m}^{2}$, and they were presented on a yellow background $\left(24 \mathrm{~cd} / \mathrm{m}^{2}\right)$, with Commission Internationale d'Eclairage (CIE) coordinates $x=0.492$ and $y=$ 0.446. All stimuli moved along one of the cardinal or oblique axes and were viewed within a square aperture rotated to align with the axis of motion. For neurophysiological experiments, aperture width was $5^{\circ}$ for neurons with foveal and parafoveal receptive fields and $10^{\circ}$ if the receptive field diameter of a neuron was $>5^{\circ}$. For human psychophysical experiments, stimuli subtended $4.7^{\circ}$ of visual angle. Three different stimulus patterns were presented in the receptive field (RF) of each neuron under study: (1) achromatic gratings, (2) heterochromatic gratings, and (3) a stimulus created by spatial superimposition of achromatic and heterochromatic gratings ("opposed-motion stimulus"). We begin by describing the characteristics of these three patterns and the procedures by which they were constructed. This is followed by a description of the manner in which they were used to assess neuronal properties.

Achromatic gratings were generated by in-phase summation of sinusoidal luminance modulations of the red and green phosphors of the display monitor. The resultant grating was uniform in chrominance ("achromatic") and appeared yellow and black at modulation peaks. Luminance contrast was set by coincident adjustments of the amplitudes of the red and green components. Drifting achromatic gratings were used to determine the preferred direction and spatiotemporal frequency for each isolated neuron.

Heterochromatic gratings were produced by counterphase summation of sinusoidal luminance modulations of the red and green phosphors. (The red and green component sinusoids were identical to those used to create achromatic gratings.) The resulting grating was of varying chrominance ("heterochromatic") and appeared red and green at modulation peaks. Luminance contrast was varied by differentially adjusting the mean luminances of the red and green component sinusoids such that the mean luminance of the resultant heterochromatic stimulus was constant across different stimulus conditions. Red and green sinusoids were set at $100 \%$ modulation depth. This $100 \%$, in essence, represents $100 \%$ of the maximal chromatic contrast obtainable from the monitor and is thus termed $100 \%$ instrument contrast. Luminance contrast of the resultant hetero- 


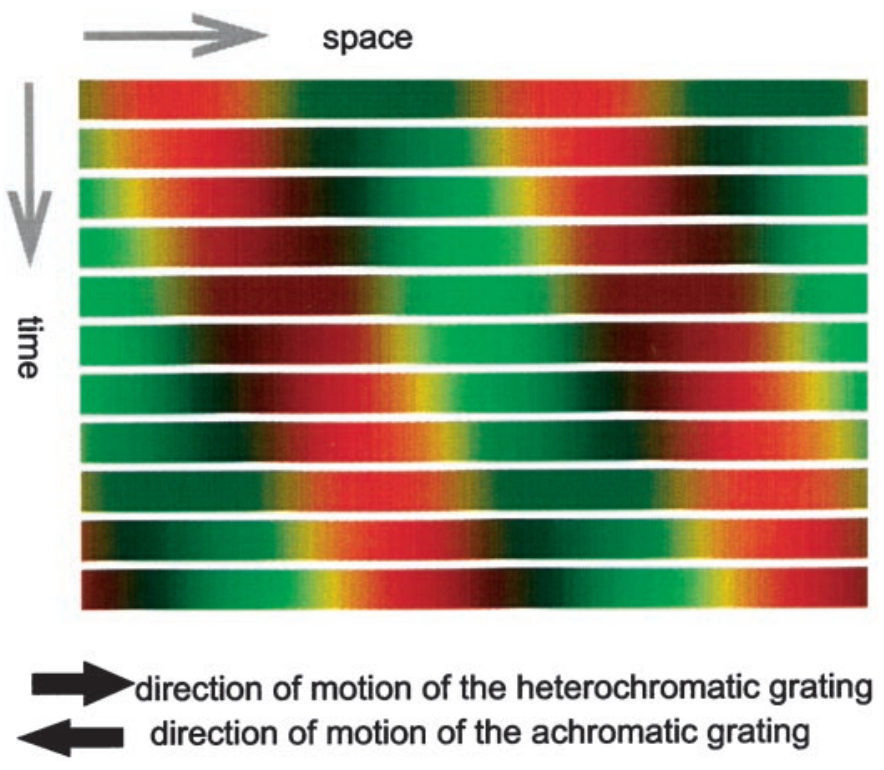

Figure 1. Space-time plot of the opposed-motion stimulus, which was formed by superposition of oppositely moving achromatic and heterochromatic sinusoids. The heterochromatic sinusoid was itself produced by antiphase summation of sinusoidal modulations of red and green display phosphors. The achromatic sinusoid was similarly produced by inphase summation of the same red and green modulations. Each horizontal slice in the space-time plot illustrates the phase relationship between achromatic and heterochromatic sinusoids for a single stimulus frame. Each frame illustrates two cycles of the compound stimulus $(0$ to $4 \times \pi)$. In this illustration achromatic and heterochromatic sinusoids were each spatially displaced by $\pi / 8$ radians (in opposite directions) between each temporal frame. The heterochromatic sinusoid in the example shown moves rightward, whereas the achromatic sinusoid moves leftward. (The yellow transition zones between red and green are attributable to reproduction and were not present in the actual stimulus.)

chromatic grating is expressed as: $\left(G_{\text {mean }}-R_{\text {mean }}\right) /\left(G_{\text {mean }}+R_{\text {mean }}\right)$. Using this metric, luminance contrast can be either positive or negative, depending on which of the two phosphor primaries is brighter with respect to human $\mathrm{V} \lambda$ isoluminance. Positive contrast refers to green more luminous than red. Likewise, negative contrast refers to red more luminous than green. The luminance contrast in these gratings was verified by measuring luminance as a function of spatial phase using a standard spot photometer (PR-650, Photoresearch).

Opposed-motion stimuli consisted of an achromatic (yellow-black) and a heterochromatic (red-green) grating, superimposed and moving in opposite directions. A space-time plot of this stimulus appears in Figure 1 . The specific configuration illustrated consists of a rightward-moving heterochromatic grating superimposed on a leftward-moving achromatic grating.

Note that when heterochromatic and achromatic gratings were combined in this manner to render an opposed-motion stimulus, the effective luminance and chromatic contrasts of each component grating were reduced by $50 \%$, whereas the mean luminance was twice the component luminance and was thus identical to the mean background luminance of $24 \mathrm{~cd} / \mathrm{m}^{2}$. (For example, the addition of an achromatic grating possessing $0 \%$ chromatic and $50 \%$ luminance contrast to a heterochromatic grating possessing $100 \%$ chromatic contrast and $0 \%$ luminance contrast brings the effective luminance contrast in the achromatic grating down to $25 \%$ and the effective chromatic contrast of the heterochromatic component down to $50 \%$ ). To facilitate comparison with the single heterochromatic and achromatic gratings, we have thus chosen to describe the opposedmotion stimuli in terms of the effective contrasts of their heterochromatic and achromatic components. In all experiments described below, the chromatic (i.e., instrument) contrast of the heterochromatic component started at $100 \%$ but was reduced to an effective chromatic contrast of $50 \%$ when added to the achromatic grating.
Wave forms of the red and green guns were as follows for the opposedmotion stimulus:

$$
\begin{aligned}
\operatorname{Red}(x, t)=R+\sin \left(2 \pi f_{\mathrm{s}} x-2 \pi f_{\mathrm{t}} t\right) & * R+\left(L / 2+\sin \left(2 \pi f_{\mathrm{s}} x\right.\right. \\
& \left.\left.+2 \pi f_{\mathrm{t}} t\right) * C * L / 2\right) * 0.41, \\
\operatorname{Green}(x, t)=G+\sin \left(2 \pi f_{\mathrm{s}} x-2 \pi f_{\mathrm{t}} t\right) & * G+\left(L / 2+\sin \left(2 \pi f_{\mathrm{s}} x\right.\right. \\
& \left.\left.+2 \pi f_{\mathrm{t}} t\right) * C * L / 2\right) * 0.59,
\end{aligned}
$$

where $x=$ spatial position, $t=$ time, $f_{\mathrm{s}}=$ spatial frequency, $f_{\mathrm{t}}=$ temporal frequency, $R$ and $G=$ mean luminances of red and green components of the heterochromatic grating, respectively, $L=$ mean luminance of the achromatic grating (fixed at $12 \mathrm{~cd} / \mathrm{m}^{2}$ for these experiments), and $C=$ luminance contrast of the achromatic grating. The factors 0.41 and 0.59 were chosen to yield a relatively neutral yellow for the achromatic stimulus with CIE coordinates $x=0.492$ and $y=0.446$.

Cone modulations. For all stimuli, cone modulations were computed from $L$ and $M$ cone excitations produced by the "red" and "green" peaks of the heterochromatic gratings. The latter were obtained by integrating the cross-product of stimulus spectral output of these stimuli by the cone fundamentals of Stockman et al. (1993). Based on these procedures, we determined that $50 \%$ chromatic contrast in our red-green gratings produced modulations of 7.74 and $-18.77 \%$ in the $L$ and $M$ cones, respectively (at $\mathrm{V} \lambda$ isoluminance). Thus, the root mean square $(\mathrm{rms}=$ square root of $\left.\left[\left(M^{2}+L^{2}\right) / 2\right]\right)$ of the independent modulations of the $L$ and $M$ cones was $14.36 \%$. For achromatic gratings, rms cone contrast was simply equivalent to Michelson luminance contrast.

\section{Stimulus paradigms for assessment of neuronal selectivity}

Direction and spatiotemporal frequency preferences. The RF of each isolated MT neuron was mapped using a black bar moving on a homogenous yellow background, which was of the same luminance and chromaticity as that used for all other measurements (see Visual stimuli: General features). Direction tuning was then assessed using an achromatic grating $\left[0.7\right.$ cycles $/^{\circ}\left(\mathrm{cyc}^{\circ}\right), 4 \mathrm{~Hz}, 100 \%$ Michelson contrast $]$ moving in each of eight different directions (along cardinal and oblique axes) centered on the RF. Directions were presented in a pseudo-random sequence for a total of five trials per stimulus type. The direction of motion that yielded the largest response was termed the "preferred" direction, and the direction opposite to the preferred was termed the "null" direction. Strength of directional bias along the preferred-null axis was quantified by a direction index (DI): $\mathrm{DI}=1-N D / P D$, where $P D$ and $N D$ are firing rate changes elicited by motion in preferred and null directions, respectively. Neurons with a DI $<0.5$ were excluded from further study.

Preferred spatial and temporal frequencies were determined for each directionally selective neuron by presenting gratings at 12 different spatiotemporal frequency combinations moving in the preferred direction (temporal frequencies, 1, 2, 4, or $8 \mathrm{~Hz}$; spatial frequencies, 0.4, 0.7, or $\left.1.4 \mathrm{cyc}^{\circ}\right)$. The spatial and temporal frequency combination yielding the largest response was used for all subsequent testing of that neuron.

Neuronal isoluminance. We defined red-green neuronal isoluminance to be the luminance contrast that elicited the smallest neuronal response to a red-green grating moving in the preferred direction. This value was determined for each MT neuron using a procedure described previously (Dobkins and Albright, 1994). Briefly, red-green heterochromatic gratings were presented at 11 different luminance contrasts, ranging in equal intervals $(4 \%)$ from $-20 \%$ (red more luminous than green) to $+20 \%$ (green more luminous than red). These stimuli were each moved in the preferred direction, and the red-green luminance contrast yielding the minimal response was identified. For subsequent EqLC experiments (see below), in which we measured neuronal responses across a range of red-green luminance contrasts, heterochromatic grating luminance contrast is expressed with respect to this neuronal isoluminance point, not relative to human $V \lambda$. For those cases in which no response minimum was found (i.e., neuronal isoluminance could not be determined), heterochromatic luminance contrast is expressed with respect to the mean red-green isoluminant point of neurons sampled (this mean was determined separately for each monkey).

\section{Equivalent luminance contrast}

Experimental design. The goal of these experiments was to quantify the sensitivity of directionally selective MT neurons to chromatic contrast. This goal was achieved using a procedure in which responses elicited by 


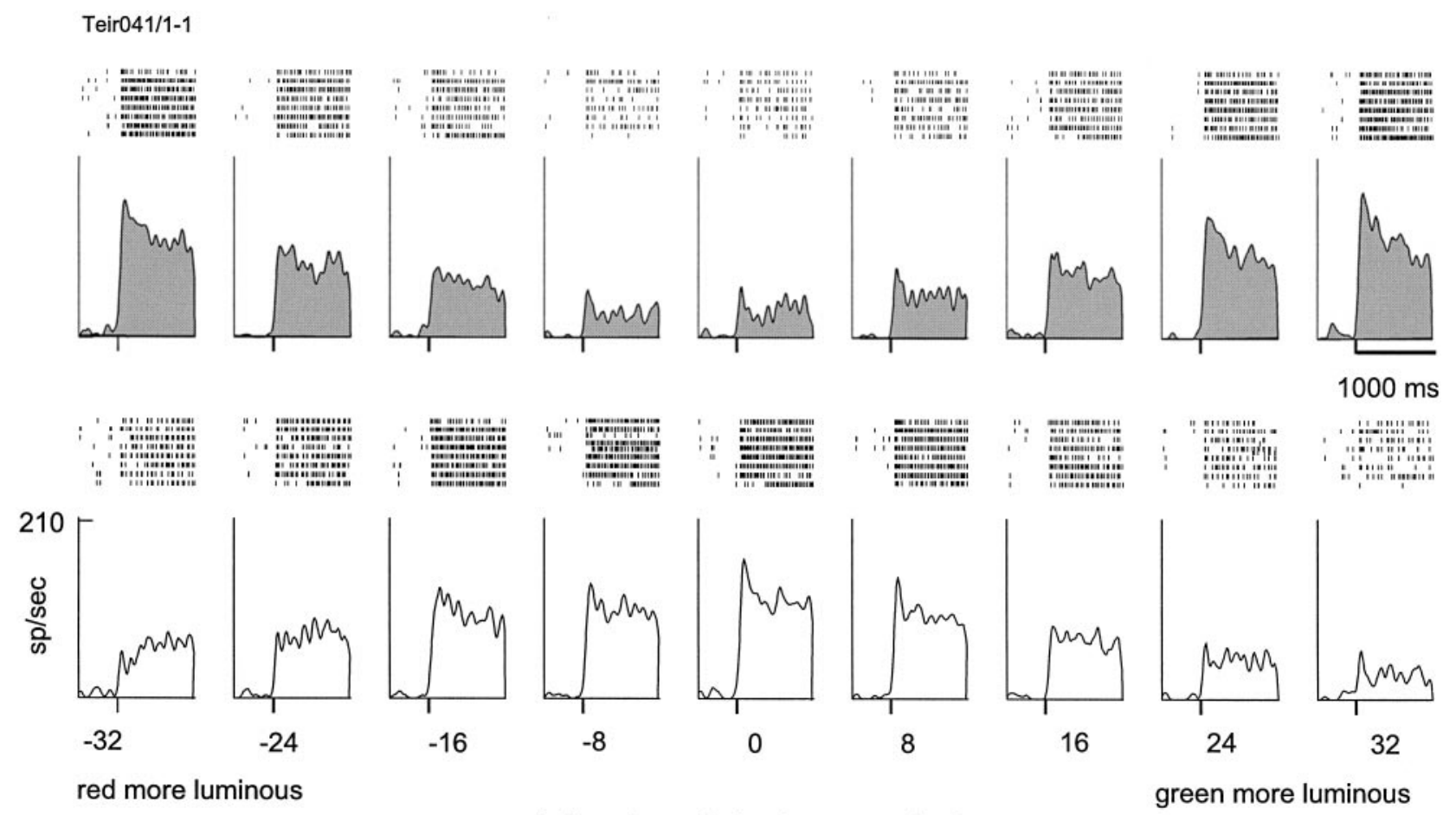

heterochromatic luminance contrast

Figure 2. Neuronal responses to the opposed-motion stimulus. Data are from a representative MT neuron, which illustrate neuronal responses as a function of (1) luminance contrast in the heterochromatic grating (columns) and (2) directional polarity of the opposed-motion stimulus (rows). The top row of rasters and peristimulus time histograms (gray shaded) illustrates neuronal responses for the directional-polarity condition in which the heterochromatic component moved in the preferred direction (whereas achromatic component moved simultaneously in the null direction). The bottom row of rasters and histograms (black outlined) illustrates responses to the corresponding luminance conditions for the inverted directional polarity. Achromatic luminance contrast was fixed at $16 \%$ for all conditions shown. Heterochromatic luminance contrast was varied from red more luminous than green (negative values on $x$-axis) to green more luminous than red. Response onset is marked at the bottom of each histogram. Stimulus duration was 1000 msec.

moving chromatically defined patterns were "balanced" against those elicited by moving luminance-modulated patterns. Neuronal sensitivity to the luminance-modulated pattern thus provided a standard against which sensitivity to chromatic contrast could be gauged, and the procedure rendered sensitivity in units of luminance contrast, i.e., the EqLC. For each MT neuron tested, EqLC was determined using the opposedmotion stimulus described above, with one component moving in the preferred direction and the other moving simultaneously in the null direction. Three independent variables were manipulated: (1) luminance contrast of the achromatic component, (2) luminance contrast of the heterochromatic component, and (3) directional polarity, such that the achromatic grating moved either in the preferred or null direction (owing to the opposed-motion configuration, the heterochromatic grating always moved conversely).

In early phases of these experiments, two achromatic contrast values were used (either 5 and 10 or 10 and $20 \%$ ); in later phases, four values were used (either $3,6,12$, and 24 or $4,8,16$, and $32 \%$ ). Luminance contrast of the heterochromatic component was varied through nine different levels, ranging from red more luminous than green to green more luminous than red through the isoluminant point of the cell (the particular range of heterochromatic luminance contrasts depended on the particular achromatic luminance contrast used). All conditions were presented in pseudo-random order.

Sample data set. The computation of EqLC was a multistep procedure that was applied to data collected using each achromatic luminance contrast value for each neuron studied. For clarity of exposition, Figures 2 and 3 illustrate the application of our procedures to a subset of data obtained from a representative neuron (the full collection of responses obtained from this neuron is presented in Fig. 5 and described in Results). The data illustrated in Figure 2 were obtained using a single achromatic contrast value. Neuronal responses are shown in the form of spike rasters and peristimulus histograms for each condition in a matrix consisting of two directional polarities (rows) and nine heterochromatic luminance contrasts (columns).

The top row of responses ( ray histograms) in Figure 2 was obtained for the directional polarity in which the heterochromatic component moved in the neuronal preferred direction, whereas the achromatic component moved in the null direction. When heterochromatic luminance contrast was high for this directional polarity, either because red was far more luminous than green, or vice versa, neuronal responses were quite large, reflecting the simple fact that the dominant heterochromatic component moved in preferred direction. Near isoluminance, however, responses were rather weak. Owing to the stimulus configuration, the latter may be a consequence of one or both of the following: (1) relative insensitivity to the motion of isoluminant stimuli, and (2) response inhibition elicited by motion of the achromatic grating in the null direction. The inhibitory contribution may be more marked when the driving power of the heterochromatic grating is small.

The bottom row of responses (white histograms) in Figure 2 was obtained for the converse directional polarity (i.e., heterochromatic component moved in null, whereas achromatic component moved in the preferred direction). This condition yielded a complimentary response pattern. Specifically, a strong response was elicited when the heterochromatic grating was isoluminant, and this response declined as the luminance contrast of the heterochromatic grating was increased.

Modeling neuronal responses. The first step in computing EqLC from a set of neuronal responses involved fitting the data by an appropriate model. We chose a Gaussian model, which was applied as follows. (The appropriateness of this model, as judged by "goodness of fit," is addressed below.) For each of the stimulus conditions presented in Figure 2 , the mean activity level was calculated over a $1000 \mathrm{msec}$ window, which began $50 \mathrm{msec}$ after stimulus onset and ended $50 \mathrm{msec}$ after stimulus offset. These activity levels are plotted correspondingly in Figure 3 (i.e., two directional polarities and nine heterochromatic luminance con- 
achromatic contrast: $16 \% \rightarrow$ EqLC $=-2.2 \%$

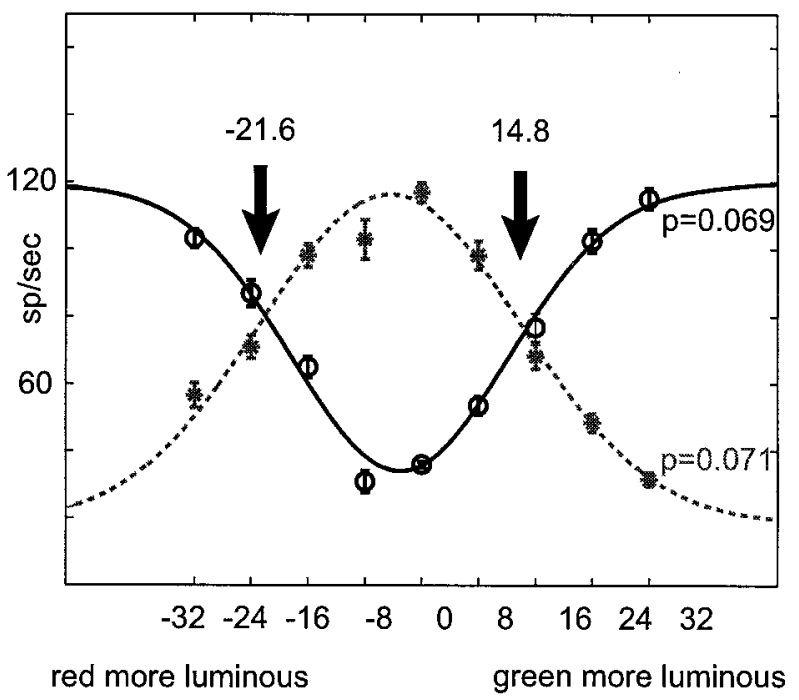

heterochromatic luminance contrast

Figure 3. Determination of equivalent luminance contrast. Mean activity levels ( $y$-axis) derived from neuronal responses shown in Figure 2 are plotted as a function of heterochromatic luminance contrast ( $x$-axis) and directional polarity (circles, asterisks). Responses obtained when the heterochromatic component moved in the neuronal preferred direction are indicated by the circles; corresponding responses obtained when the achromatic component moved in the preferred direction are indicated by asterisks. Achromatic luminance contrast was fixed at 16\%. Motion "null points" were defined to be the heterochromatic luminance contrast values for which the neuron lost sensitivity to directional polarity. In practice, these null points can be identified graphically by the intersections (arrows) of the two Gaussian-fitted directional-polarity response functions. For this data set, null points occurred at luminance contrasts of $-21.6 \%$ (red more luminous than green) and $14.8 \%$ (green more luminous than red). EqLC was computed as the difference between (1) the average of the absolute values of these null points and (2) the achromatic luminance contrast used to construct the opposed-motion stimulus. EqLC thus reflects sensitivity to the chromatic contrast in the stimulus calibrated in units of luminance contrast.

trasts), along with SEs (some of which are hidden by the symbols). Gaussians were then fitted to the set of responses elicited by the ninecontrast levels, separately for the each of the two directional polarities, using the following functions:

$$
\begin{aligned}
& f(x)=a+b * \exp ^{-c *(x-\mu) \wedge 2}, \\
& f(x)=a-b * \exp ^{-c *(x-\mu) / 2},
\end{aligned}
$$

The choice of Equation 3 versus 4 depended on directional polarity, such that the former was used when the achromatic grating component moved in the preferred direction, and the latter was used when it moved in the null direction. Here, $a$ corresponds to the minimum (maximum) of the tuning curve, $b$ corresponds to response differential relative to the minimum (maximum), $c$ corresponds to width, and $\mu$ corresponds to the value of heterochromatic luminance contrast yielding the response minimum (maximum).

The solid line in Figure 3 represents the fit to data obtained when the heterochromatic component moved in the preferred direction; the dashed line represents the fitted curve for data obtained when the achromatic component moved in the preferred direction.

The appropriateness of the Gaussian model was evaluated for each data set on the basis of maximum likelihood estimates of the fitted parameters, which were obtained by minimizing $\chi^{2}$ (Press et al., 1992). In this procedure, $\chi^{2}$ corresponds to the sum of squared errors between model predictions and data, divided by the SD of the experimental measurements. Because the SDs of neuronal firing rate may be non- normally distributed, we minimized $\chi^{2}$ by dividing by the SE. This procedural modification yields more conservative estimates of goodness of fit. We used this procedure to model a minimum of eight data sets [four achromatic luminance contrast conditions $\times$ two directional-polarity conditions] for each cell. Cells were excluded from further analysis if the $Q$ value (indicative of goodness of fit; Press et al., 1992) for any data set obtained from a neuron was $<0.01$. This ensured that reliable EqLCs could be derived.

Determining the motion null points. The luminance conditions for which a neuron is equally sensitive to the achromatic and heterochromatic components of the opposed motion stimulus are defined by loss of neuronal sensitivity to the directional polarity of the stimulus (i.e., when equivalent responses are elicited regardless of whether the heterochromatic or achromatic component of the stimulus moves in the preferred direction). We term these luminance conditions the "motion null points," and they were derived from the intersections (Fig. 3, arrows) of the curves fitted to the responses obtained using the two directional polarities. As for the data in Figure 3, under most (but not all; see Results) conditions, we observed two null points for each data set: one to each side of isoluminance (denoted as $0 \%$ luminance contrast). Intuitively, this simply means that a null point can be obtained when red is brighter than green and vice versa.

Computing equivalent luminance contrast. EqLC was computed as the difference between the average null point (the mean of the absolute values of the two heterochromatic luminance contrasts eliciting a null) and the luminance contrast of the opposing achromatic grating:

$$
\mathrm{EqLC}=C_{\text {achro }}-\left(\left|C_{\text {hetchro }} 1\right|+\left|C_{\text {hetchro }} 2\right|\right) / 2,
$$

In Equation $5, C_{\text {achro }}$ corresponds to achromatic luminance contrast; $C_{\text {hetchro }} 1$ and $C_{\text {hetchro }} 2$ refer to the two heterochromatic luminance contrasts that yielded motion nulls. The EqLC value obtained by this procedure is the average luminance contrast difference between the opposing heterochromatic and achromatic gratings that renders a motion null and is thus a calibrated measure of neuronal sensitivity to the heterochromatic component.

The EqLC procedure illustrated for this sample data set was repeated for additional data sets from each neuron studied, which were obtained using preselected values of achromatic luminance contrast. This allowed us to investigate whether EqLC varies as a function of achromatic luminance contrast. For those neurons tested with four achromatic luminance contrast values, this protocol resulted in 96 conditions (64 EqLC conditions, 32 single gratings). We attempted to record 10 trials per condition. Neurons with as few as five trials per condition were included in our sample.

\section{Human psychophysical experiments}

Our quantification of the sensitivity of MT neurons to moving heterochromatic patterns was primarily motivated by a desire to determine whether this neuronal sensitivity could account for perceptual sensitivity to such stimuli. Although there have been numerous reports of the sensitivity of human subjects to moving heterochromatic patterns, including one study that used an opposed-motion stimulus (Cavanagh and Anstis, 1991), we thought it critical that the neuronal-perceptual comparison be made on the basis of results obtained under conditions that were as similar as possible.

We therefore measured EqLC in two human observers. In these psychophysical experiments, all stimulus conditions and display methods were identical to those used in our MT study (see above), with the exception that we obtained data for only one spatiotemporal frequency; values used $\left(0.7 \mathrm{cyc} /{ }^{\circ}, 4 \mathrm{~Hz}\right)$ reflected the preference of the majority of MT neurons sampled. Stimuli were viewed from a distance of $53 \mathrm{~cm}$, and they were positioned within a square $\left(4.7 \times 4.7^{\circ}\right)$ window that was centered $3.7^{\circ}$ eccentric to the point of fixation (the average receptive field eccentricity for our sample of MT neurons) along the horizontal meridian in the right visual hemifield. All stimuli were located at the center of the display monitor; visual field location was determined by appropriate positioning of the $0.2^{\circ}$ square fixation on the display monitor.

Three independent variables were manipulated systematically: (1) luminance contrast of the achromatic component, (2) luminance contrast of the heterochromatic component, and (3) directional polarity (stimulus motion was along the horizontal axis). Luminance contrast of the achromatic component was pseudo-randomly selected from one of five predetermined values $(10,15,20,25$, and $30 \%)$. Luminance contrast of the heterochromatic component was varied through 19 different levels in 
a pseudo-random sequence (the particular range of heterochromatic luminance contrasts depended on the particular achromatic luminance contrast used). Directional polarity of the opposed-motion stimulus was varied randomly across trials, and psychophysical data were averaged across the two polarity values.

Subjects were required to fixate for the duration of each stimulus presentation. Eye position was monitored during initial phases of testing, and we confirmed that fixation was accurate $\left( \pm 1^{\circ}\right)$ and direction of eye movement was not systematically related to stimulus conditions or the behavioral choice. These observations are consistent with previous reports that human subjects are capable of reliable fixation under similar stimulus conditions (Murphy et al., 1975). Stimuli were presented in a two-alternative forced-choice paradigm in which the dependent variable was perceived direction of motion, as assessed by a key press at the end of each trial. For each of the 19 heterochromatic luminance contrasts, the proportion of trials in which motion was perceived in favor of the direction of motion of the achromatic grating was determined. These data were fitted with Weibull functions. As for neuronal data, psychophysical null points corresponded to the loss of sensitivity to directional polarity, which was manifested in this paradigm by chance $(50 \%)$ performance on the direction discrimination task. Two null points were generally seen for each block of 19 heterochromatic luminance contrasts: one for a condition in which green was more luminous that red and a second when red was more luminous than green. EqLC was derived using Equation 5. The same procedure was repeated for each achromatic luminance contrast such that a total of five EqLC values were obtained from each subject.

\section{RESULTS}

\section{Basic response properties}

Data from 95 MT neurons in two monkeys were included in this study. The receptive field centers, direction preferences, direction indices, and spatiotemporal tuning of these neurons are shown in Figure 4 . The vast majority of cells possessed parafoveal receptive fields, with a mean eccentricity of $3.7 \pm 1.6^{\circ}$ (Fig. $4 A$ ). The distribution of preferred directions was approximately uniform, and the mean direction index across neurons was $1.06 \pm 0.56$ (Fig. $4 B)$. The distribution of preferred spatiotemporal frequencies (Fig. 4C) demonstrates that, on average, MT neurons preferred temporal frequencies in the range of 4 and $8 \mathrm{~Hz}$ rather than 1 and $2 \mathrm{~Hz}$ (mean, $4.9 \mathrm{~Hz}$ ). The distribution of preferred spatial frequency, by contrast, was relatively uniform (mean, $0.81 \mathrm{cyc} /{ }^{\circ}$ ).

\section{Equivalent luminance contrast}

\section{Representative neuronal responses}

A very consistent pattern of responses to the opposed-motion stimulus was observed across the population of cells tested. Data from a representative neuron are illustrated in Figure 5. This figure contains the complete collection of responses to the opposed motion stimulus obtained from the neuron presented above (see Materials and Methods and Figs. 2, 3 where the responses were used to illustrate procedures for computing EqLC). The data were obtained using four different achromatic luminance contrasts $(4,8,16$, and $32 \%$; Fig. $5 A-D)$, nine heterochromatic luminance contrasts (columns within each panel), and two directional polarities (rows within each panel).

At $4 \%$ achromatic luminance contrast (Fig. $5 A$ ), the two directional-polarity curves failed to intersect, reflecting the dominance of the heterochromatic grating under all conditions tested. Because no null points were measured, we can only place a lower boundary on our estimate, such that EqLC $\geq 4 \%$ (bounded by the achromatic luminance contrast used for these data). When achromatic luminance contrast was increased, EqLC declined. For achromatic luminance contrast values of $8 \%$ (Fig. $5 B$ ), 16\% (Fig. $5 C$ ), and $32 \%$ (Fig. 5D), the two directional-polarity curves intersected at two points, one on either side of the neurons isoluminance point. From these data, EqLC was computed to be
$1.5,-2.2 \%$, and $-3.3 \%$ for achromatic luminance contrast values of 8,16 , and $32 \%$, respectively, indicative of a lessening influence of the chromatic stimulus component on the cell response.

We were able to determine EqLC for 95 of 125 (76\%) of the cells that were tested with either two or four different achromatic luminance contrasts. For the remaining neurons, EqLC could not be determined, owing to at least one of the following reasons: (1) all values of achromatic luminance contrast used were insufficient to null the heterochromatic component; (2) directional selectivity (DI $>0.5$ ) was not present at low luminance contrast; (3) no responses were elicited by any of the opposed-motion stimuli; or (4) the Gaussian fit had to be rejected. When a neuron failed to exhibit null points at a subset of achromatic luminance contrasts (as was the case for the conditions illustrated in Fig. 5A), we determined a lower boundary estimate of EqLC. In such cases, EqLC was lower-bounded by the larger of (1) the value of EqLC obtained for the next higher level of achromatic luminance contrast used or (2) the value of the achromatic luminance contrast for which no null point was observed.

\section{Population data: general characteristics}

To obtain a better impression of overall activity in our neuronal sample in response to the opposed-motion stimulus, we plotted the average neuronal response as a function of heterochromatic luminance contrast separately for each achromatic luminance contrast (Fig. 6A). Individual neuronal isoluminant points were aligned before averaging across the population, such that the $x$-axis reflects "relative" luminance contrast (with respect to the neural isoluminant point) in the heterochromatic grating. The population data were fitted with the same functions used to fit data from single neurons (Eqs. 3 and 4). The data shown in Figure $6 A$ are from 20 neurons, which were selected for this analysis because all were tested under identical luminance contrast values. (Although additional data are omitted from this figure for clarity of illustration, a qualitatively and quantitatively similar pattern of results was observed for neurons tested under different luminance contrast values.) As was the case for the representative neuron illustrated in Figure 5, responses were dominated by the heterochromatic grating when achromatic luminance contrast was low ( $<8 \%$; Fig. $6 A$, top panels $)$. This population heterochromatic dominance indicates that the degree of red-green input to MT neurons is quite strong and even overrides luminance contrast signals up to at least 4\%. As for single-neuron data, the population EqLC decreased with increasing luminance contrast in the achromatic grating. This finding indicates that processing of moving stimuli defined by luminance and chromatic contrast is slightly less effective compared with stimuli defined by luminance contrast only. However, the former are still effectively processed by the motion system.

The data we have obtained using the opposed-motion stimulus are a subsample from the space of possible achromatic and heterochromatic luminance contrast combinations. Motion null points (hence EqLC) for any combination of heterochromatic and achromatic stimuli within this space can be predicted from our data by (1) computing the difference between the two directional-polarity average response functions (from Fig. 6A) for each achromatic luminance contrast and (2) interpolating between computed difference curves along the dimension of achromatic luminance contrast. The outcome of this process is illustrated in Figure $6 B$. The heterochromatic and achromatic luminance contrast dimensions are represented along the $x$ - and $y$-axes, respectively. The two converging diagonals (dashed black lines) within 
A

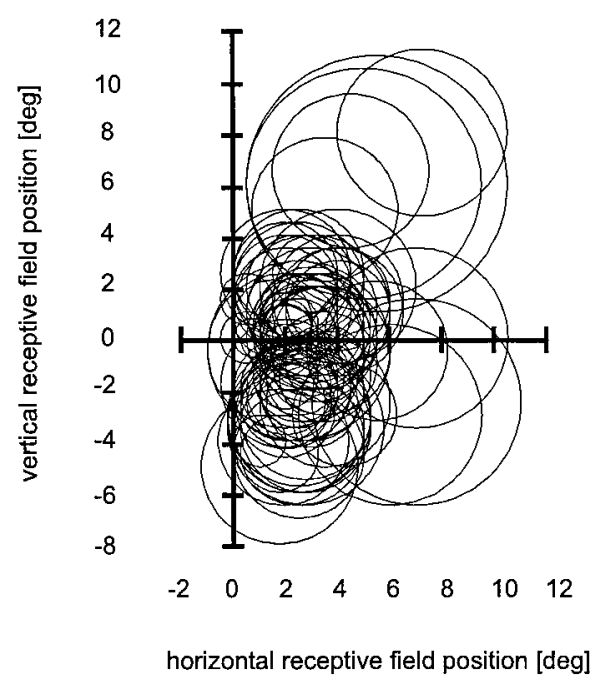

B

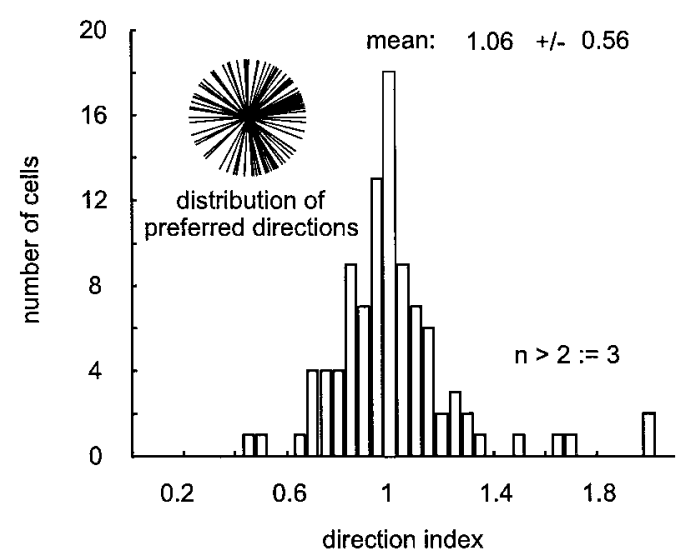

C

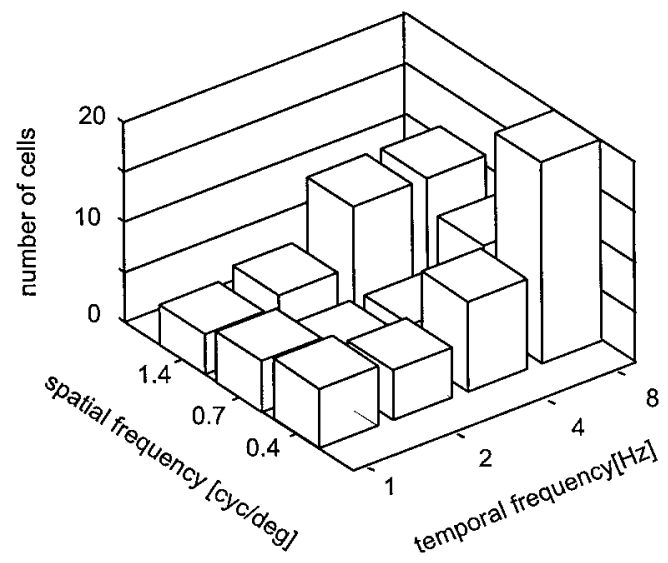

Figure 4. General response characteristics of the sample neurons. A, Receptive field location and diameter for each of the neurons. The $x$-axis represents the azimuth of the visual field (degrees); the $y$-axis represents elevation. The majority of receptive field centers were located within $5^{\circ}$ of the center of gaze. $B$, Distribution of direction indices and preferred directions of motion (inset). Most cells were highly directional, and the distribution of preferred directions was uniform. $C$, Distribution of preferred spatiotemporal frequencies. Bar height indicates the number of cells as a function of preferred temporal ( $x$-axis) and spatial ( $y$-axis) frequency. this space indicate points of identical luminance contrast for achromatic and heterochromatic components. The directionalpolarity response difference is indicated by a gray scale (right panel ). Darker intensities indicate that the heterochromatic component dominated the response, whereas lighter intensities indicate that the achromatic component dominated. The solid white line is the nulling contour (response difference, 0 ), which indicates conditions that will render the average MT neuron insensitive to the directional polarity of the opposed-motion stimulus. Inasmuch as the nulling contour deviates from the same-contrast diagonals, the average neuron exhibits sensitivity to the chromatic contrast in the stimulus. Likewise, from the difference between these diagonals and the nulling contour measured along either axis, the plot allows one to predict EqLC for any set of conditions within this space.

\section{Relationship between EqLC and luminance contrast in the achromatic grating}

The representative neuron in Figure 5 and the subset of population data illustrated in Figure 6 reveal a consistent tendency for EqLC to decline with increasing achromatic luminance contrast. Indeed, this relationship was observed in 79 of $95(83.1 \%)$ of the sampled cells. The EqLC of 13 of the remaining 16 cells remained approximately constant for different luminance contrasts [range tested, 10 and $20 \%(n=5$ of 35$) ; 3-24 \%(n=4$ of 18$)$; and $4-32 \%(n=4$ of 42$)]$ and was observed within the range of $\sim 2-6 \%$ for all achromatic stimulus conditions used. An example of such a neuron is shown in Figure 7. This MT neuron was tested at achromatic luminance contrasts of $4,8,16$, and $32 \%$, and the respective EqLCs were $\sim 4,6.8,4.1$, and 3.5\%. Thus, although it exhibited the same response characteristics to the opposedmotion stimulus as the neuron illustrated in Figure 5, EqLC remained relatively constant across values of achromatic luminance contrast. For the remaining three cells, EqLC increased slightly as a function of luminance contrast.

A previous report (Gegenfurtner and Hawken, 1995) provided evidence that chromatic sensitivity to motion is substantially different for foveal versus parafoveal presentation. In view of this evidence, we considered the possibility that neurons with relatively invariant values of EqLC have more foveally located receptive fields than those for which EqLC declined with increasing luminance contrast. The mean eccentricity of neurons expressing relatively invariant values of EqLC was $3.2^{\circ}$, whereas that of neurons for which EqLC declined with increasing luminance contrast was $3.9^{\circ}$. This difference was not significant ( $p=0.131$, $t$ test), suggesting that the EqLC-luminance relationship is unrelated to the previously reported functional distinction associated with receptive field eccentricity.

A more complete picture of the observed relationships between EqLC and achromatic luminance contrast for individual neurons can be seen in Figure 8. Plotted is the EqLC as a function of luminance contrast (for the subset of neurons for which a complete set of data were obtained at each of the following achromatic contrasts: $4,8,16$, and $32 \%$ ). Cells were placed in one of two categories based on the observed relationship between EqLC and achromatic contrast. The first category (Fig. 8, left) consists of those cells for which EqLC declined with increasing luminance contrast. Neurons were placed in this class if the EqLC obtained at the highest luminance contrast was no more than $60 \%$ of that obtained at the lowest luminance contrast. The second category (Fig. 8, right) consisted of those cells for which EqLC was relatively invariant over changes in luminance contrast. 

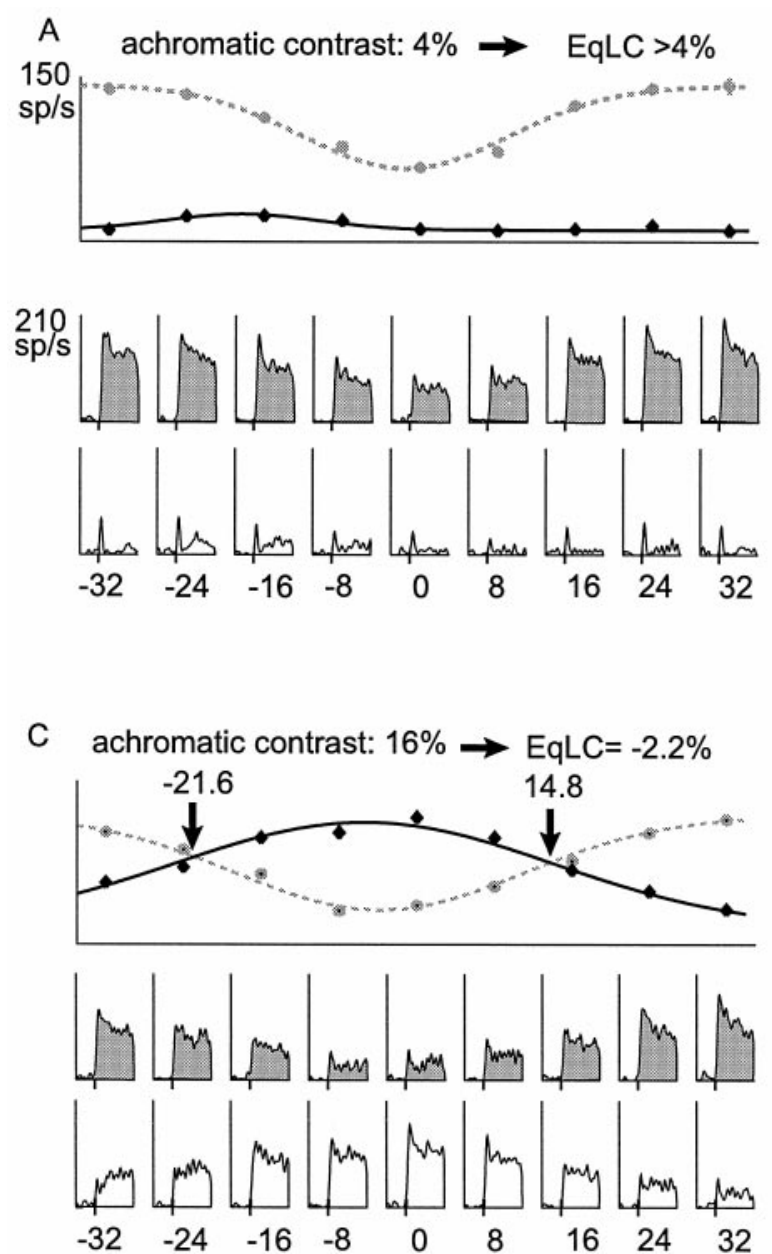

teir041/1-1
B
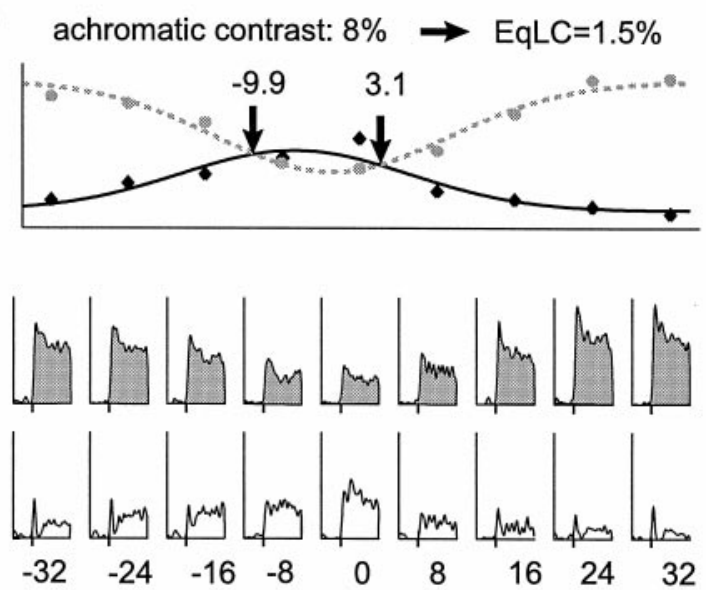

D
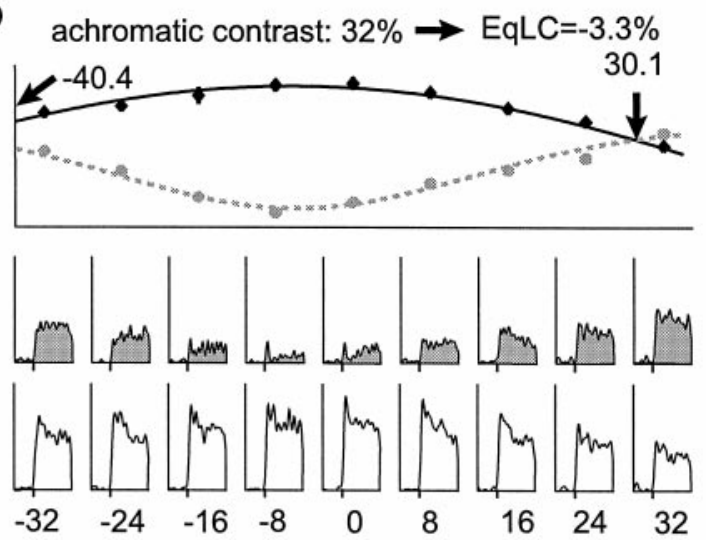

luminance contrast in heterochromatic grating [\%]

Figure 5. EqLC response pattern I: characteristic response pattern of an MT neuron to the opposed-motion stimulus. The EqLC for this neuron was determined at four different luminance contrasts $(4,8,16$, and $32 \%)$. The four quadrants $(A-D)$ illustrate data obtained under these four conditions. Each quadrant contains two rows of cumulative histograms (bottom) and a graph of mean responses (top). The top gray shaded row of histograms in each quadrant shows neuronal activity obtained for one of the two directional-polarity conditions: heterochromatic component moved in the preferred direction. The bottom row of histograms shows neuronal activity for the opposite directional polarity: achromatic component moved in the preferred direction. Within each row of histograms, data are shown for each of nine different values of heterochromatic luminance contrast $(-32$ to $32 \%$ in $8 \%$ increments; negative contrast values correspond to red more luminous than green and vice versa for positive values). Mean responses are plotted at top. Data from each of the two directional-polarity conditions have been fitted with a Gaussian. The gray line and symbols represent results for the condition in which the heterochromatic component moved in the neuronal preferred direction. The black line and symbols represent results for the condition in which the achromatic component moved in the preferred direction. (Note that when either component moved in the preferred direction, the other moved simultaneously in the anti-preferred direction.) For this neuron, EqLC decreased with increasing luminance contrast, becoming effectively negative at high contrast. This inverse relationship was characteristic of the vast majority of MT neurons studied. See Results for details. Calibration $(C$, bottom) indicates stimulus onset and period of stimulus presentation $(1000 \mathrm{msec})$. Prestimulus time was $500 \mathrm{msec}$.

For further evaluation, we provide in Table 1 an inventory of the number of neurons tested at different achromatic contrasts, the number of neurons for which EqLC could be determined, as well as the mean, SE, and median EqLC.

To evaluate the conjoint behavior of the whole-cell sample, EqLC was averaged across all 95 neurons studied and plotted as a function of luminance contrast in the achromatic grating, separately for each of two monkeys (Fig. 9A). For both animals, mean EqLC was $\sim 5 \%$ when achromatic contrast was low $(<10 \%)$, decreased with increasing achromatic contrast amplitude, and became negative when achromatic contrast exceeded $\sim 17-23 \%$. The results from a Kruskal-Wallis ANOVA revealed that the decline of EqLC was significant for both monkeys ( $p<$ 0.0001).

For the foregoing analyses we computed and expressed EqLC as a function of the luminance contrast in the opposing achromatic grating. To fully appreciate the contribution of color to motion processing, one would also like to know how chromatic motion sensitivity, as assessed by EqLC, depends on luminance contrast in the same moving component. A simple recalculation allowed us to express our data in this manner. Recall that EqLCs were empirically determined as the difference between the lumi- 

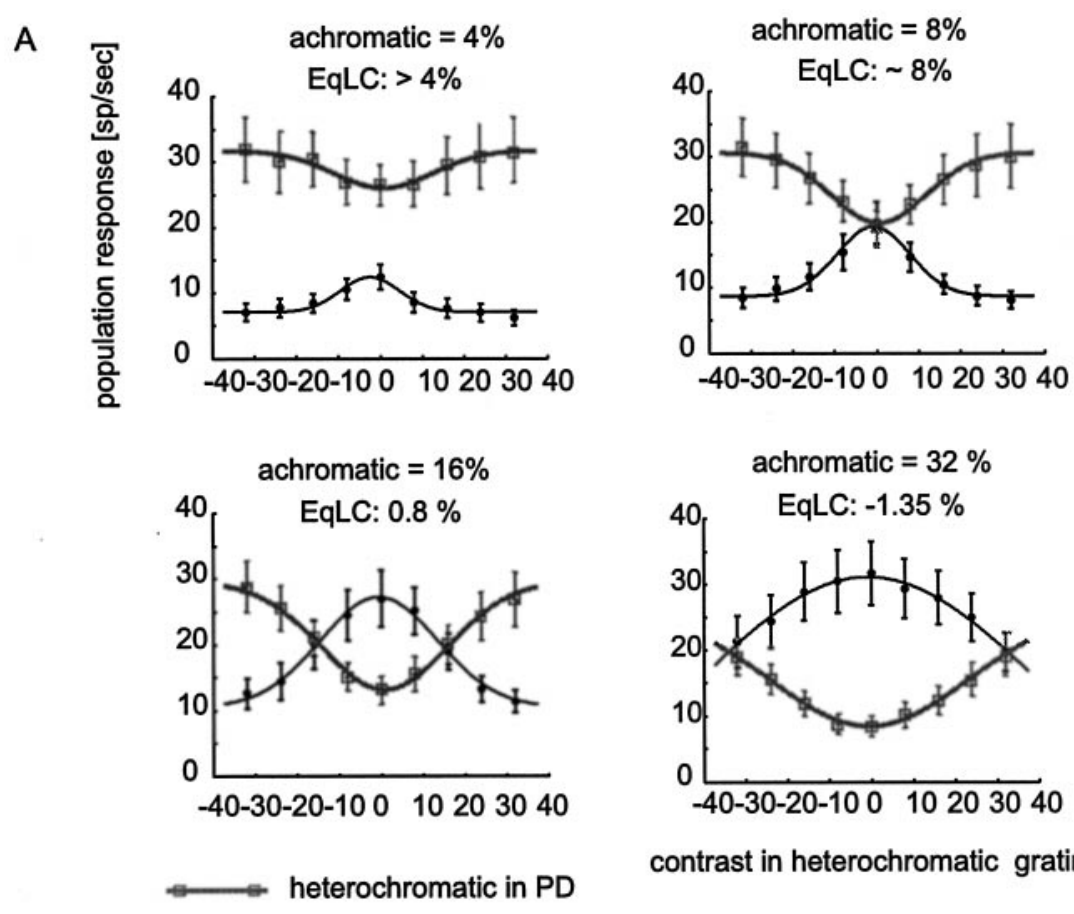

contrast in heterochromatic grating [\%]

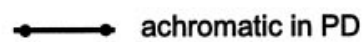

B
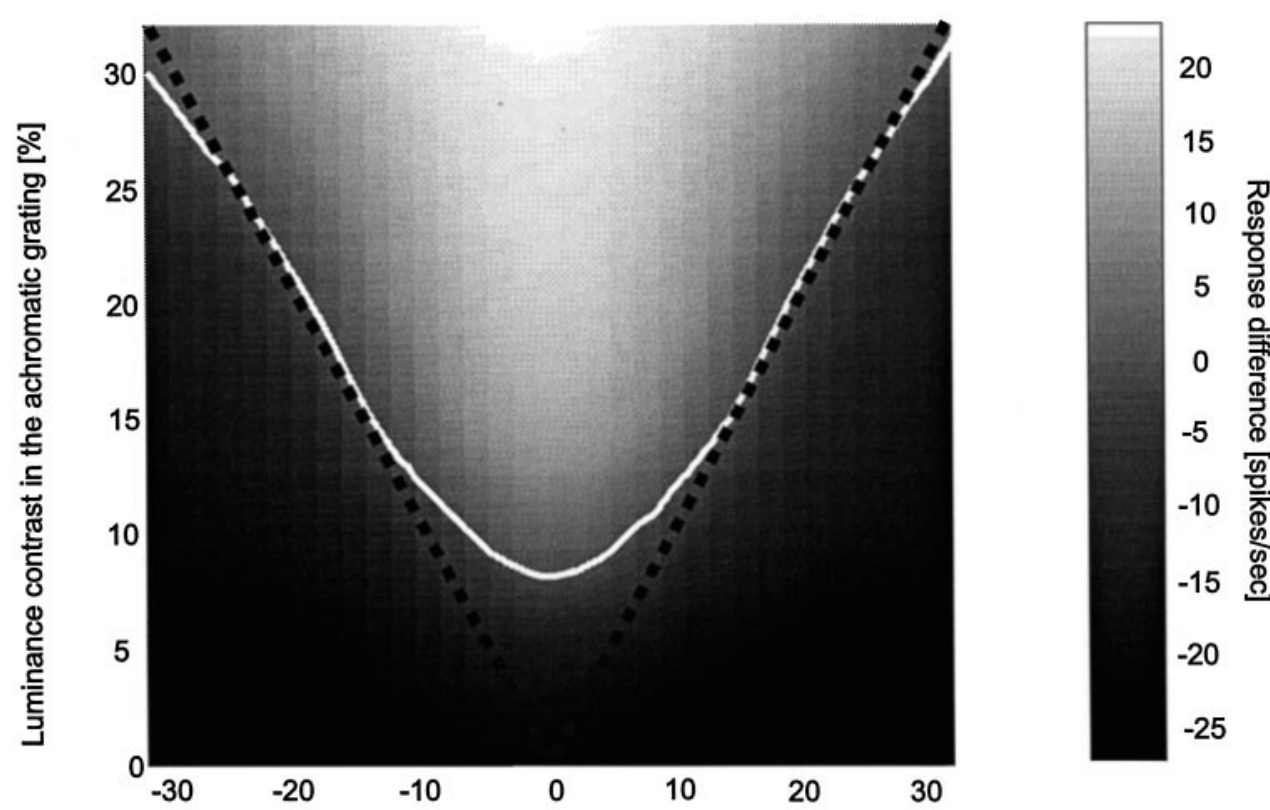

Luminance contrast in the heterochromatic grating [\%]
Figure 6. Average MT activity: average neuronal activity as a function of achromatic contrast is shown for a sample of neurons tested under identical stimulus conditions $(n=20)$. $A$, EqLC was determined for each neuron in this subsample using an opposed-motion stimulus constructed from each of four achromatic gratings, which differed solely in the amplitude of luminance contrast $(4,8,16$, and $32 \%$ ). The four quadrants illustrate data obtained under these four conditions, which have been averaged over all 20 cells. Each quadrant contains a plot of mean responses as a function of heterochromatic luminance contrast, which ranged from -32 to $32 \%$ in $8 \%$ increments. Error bars indicate SE for each data point. The two curves in each plot (gray and black) have been fitted to data from each of the two directional-polarity conditions. Motion null points for each achromatic luminance contrast are defined by loss of sensitivity to directional polarity and are identifiable graphically by intersections of the two directional-polarity curves. Average EqLC values computed from these null points decreased with increasing luminance contrast, becoming negative at high contrast. ( $B)$ Our experiments subsampled the relevant space of achromatic and heterochromatic luminance contrasts. The regularity of our data allows one to predict EqLC for any combination of contrast values within this space by (1) computing the difference between the two directional-polarity average response functions (from $A$ ) for each achromatic luminance contrast and (2) interpolating between computed difference curves along the dimension of achromatic luminance contrast. The resulting directional-polarity difference values (gray scale) have been plotted as a function of heterochromatic and achromatic luminance contrast, which are represented along the $x$ - and $y$-axes, respectively. Dark shading indicates that the heterochromatic component dominated the response, whereas light shading indicates that the achromatic component dominated. The two converging diagonals (dashed black lines) within this space indicate points of identical luminance contrast for achromatic and heterochromatic components. The solid white line is the nulling contour (response difference, 0), which indicates conditions that will render the average MT neuron insensitive to the directional polarity of the opposed-motion stimulus. Inasmuch as the nulling contour deviates from the same-contrast diagonals, the average neuron exhibits sensitivity to the chromatic contrast in the stimulus. Likewise, from the difference between these diagonals and the nulling contour measured along either axis, the plot allows one to predict EqLC for any set of conditions within this space. See legend to Figure 5 and Results for details. 

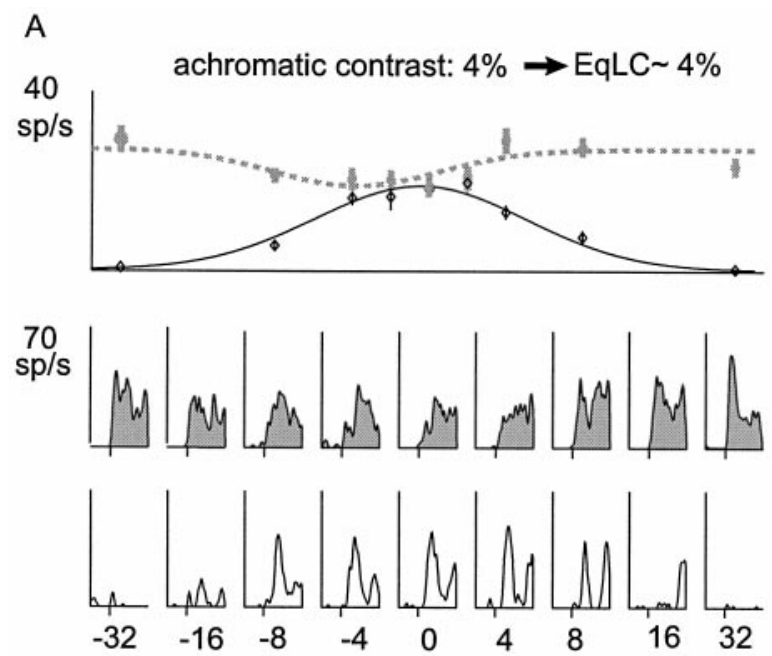

C
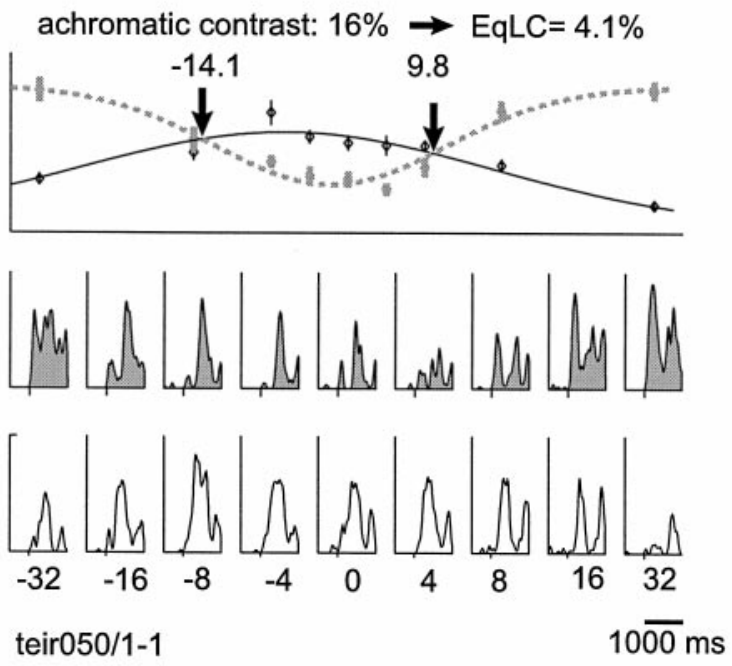

B
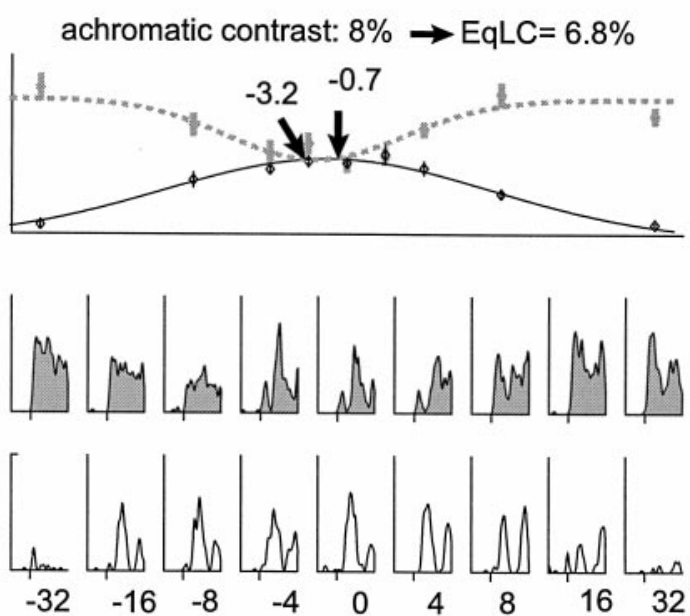

$\mathrm{D}$
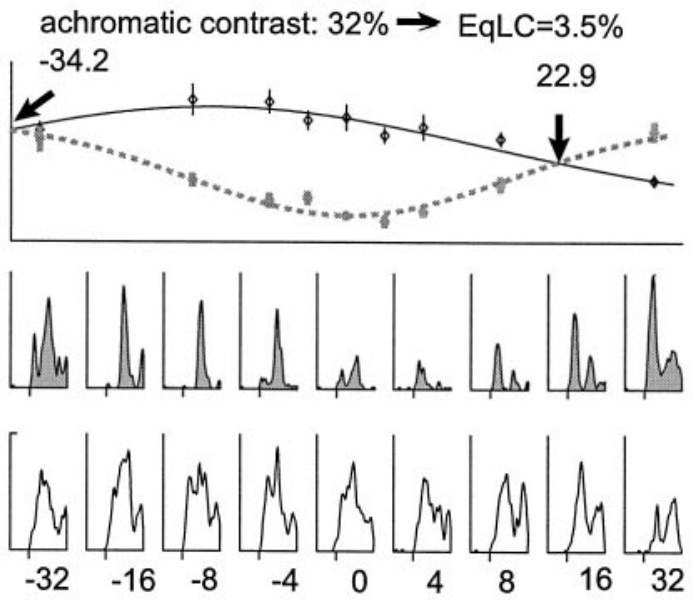

luminance contrast in heterochromatic grating [\%]

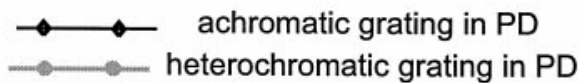

Figure 7. EqLC response pattern II: example of an MT neuron for which EqLC was found to be independent of luminance contrast. The EqLC for this neuron was determined using an opposed-motion stimulus constructed from each of four achromatic gratings, which differed solely in the amplitude of luminance contrast $(4,8,16$, and $32 \%)$. The four quadrants of the figure illustrate data obtained under these four conditions. For this neuron, EqLC was $\sim 4 \%$ for all achromatic luminance contrasts tested. Plotting conventions are as in Figure 5. Also see legends to Figures 2, 3, and 5, Materials and Methods, and Results for details.

nance contrasts in achromatic and heterochromatic components that gave rise to motion nulls (i.e., EqLC = achromatic luminance contrast - heterochromatic luminance contrast null $_{1}$ ). Therefore, subtracting EqLC from achromatic luminance contrast yields heterochromatic luminance contrast for the same EqLC. These values are plotted in Figure $9 B$. The pattern of results indicates that the contribution of red-green chromatic contrast to the neuronal response to a moving pattern is heavily dependent on, and inversely proportional to, the degree of luminance contrast in the same pattern. When luminance contrast is low, red-green chromatic contrast exerts a significant positive influence over the neuronal response. When luminance contrast is relatively high (more than $\sim 15 \%$ ), coincident red-green chromatic contrast is of little consequence or may actually have a negative effect on sensitivity to the moving pattern.

\section{Relationship between EqLC and preferred spatial and temporal frequency}

It has been shown previously that psychophysically determined EqLC in human subjects varies significantly as a function of temporal and spatial frequency (Cavanagh and Anstis, 1991). The range of spatial and temporal frequencies investigated in this study is a superset of those investigated by Cavanagh and Anstis (1991), and therefore this is an additional parameter that allows us to compare psychophysical and neuronal data. Mean EqLC is plotted in Figure $10 A$ as a function of achromatic luminance contrast ( $x$-axis) and temporal frequency (individual curves) for neurons tested at four different achromatic contrasts. (Data from neurons preferring 1 and $2 \mathrm{~Hz}$ have been pooled, because there were relatively few neurons selective for these low frequencies). A 

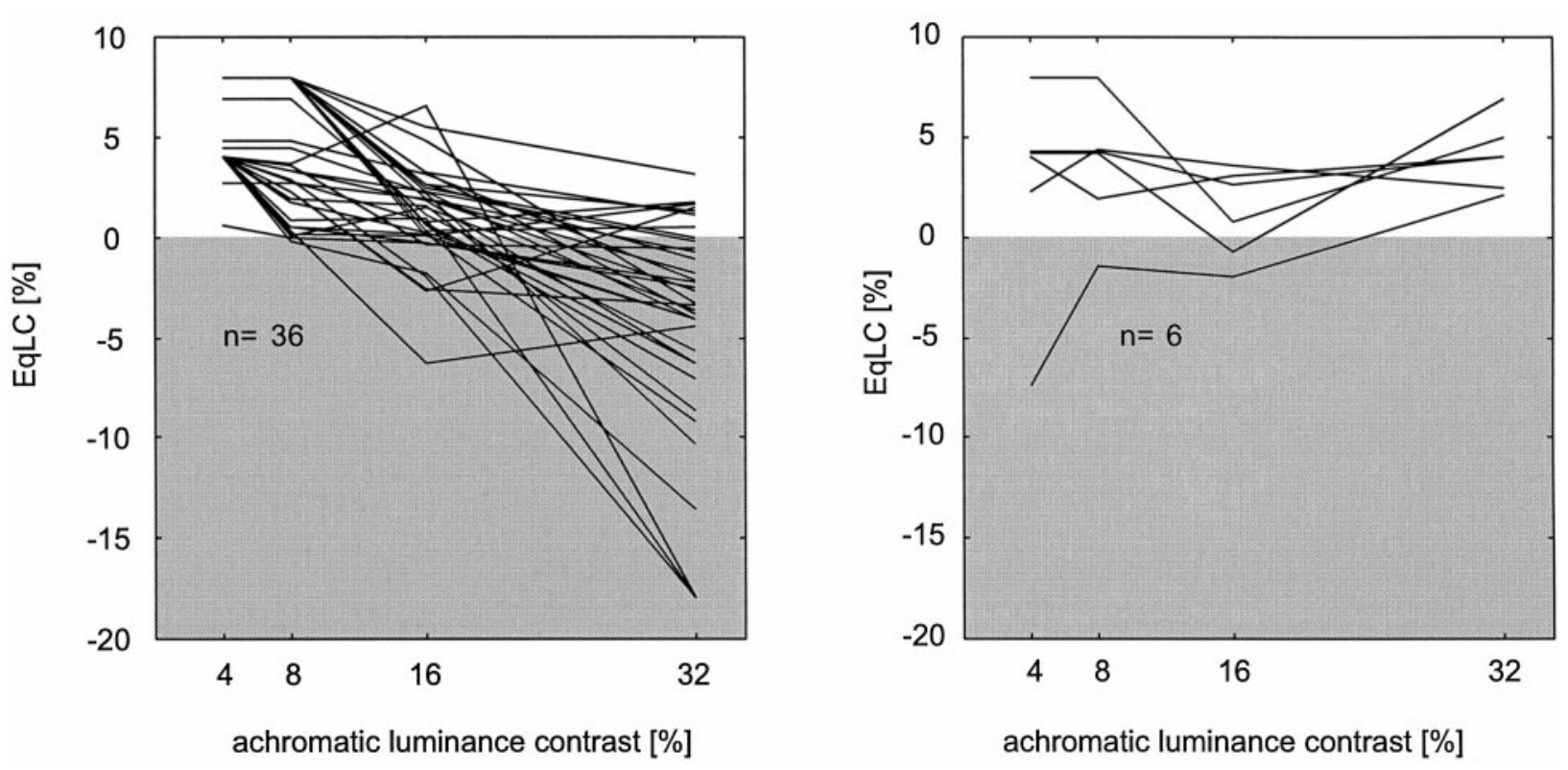

Figure 8. EqLC of individual neurons as a function of luminance contrast. $A$, Neurons that showed a decline of EqLC (i.e., their EqLC at the highest achromatic contrast was no more than $60 \%$ of the EqLC at the lowest achromatic contrast). The EqLCs at different achromatic luminance contrast for a given neuron are connected by straight lines. Plotted are those neurons for which EqLC was determined at 4, 8, 16, and 32\% achromatic luminance contrast. $B$, Neurons that did not show a systematic decrease of EqLC as luminance contrast increased. As in $A$, only neurons whose EqLC was determined at $4,8,16$, and $32 \%$ achromatic luminance contrast are plotted.

\begin{tabular}{|c|c|c|c|c|}
\hline Monkey T & $5 \%$ contrast & $10 \%$ contrast & $20 \%$ contrast & \\
\hline$n=7(9)$ & $4.72(5.0)$ & $3.01(3.15)$ & & \\
\hline$n=33(40)$ & & $3.54 \pm 7.68(3.3)$ & $-1.12 \pm 11.85(0.7)$ & \\
\hline Monkey K & $3 \%$ contrast & $6 \%$ contrast & $12 \%$ contrast & $24 \%$ contrast \\
\hline$n=18(22)$ & $4.63(6.0)$ & $5.04(6.0)$ & $1.49 \pm 1.54(1.6)$ & $-1.07 \pm 5.26(-0.05)$ \\
\hline Monkey $\mathrm{T}$ & $4 \%$ contrast & $8 \%$ contrast & $16 \%$ contrast & $32 \%$ contrast \\
\hline$n=19(28)$ & $4.78(4.0)$ & $4.0(4.1)$ & $0.76 \pm 2.84(1.2)$ & $-1.89 \pm 5.02(-1.2)$ \\
\hline Monkey K & $4 \%$ contrast & $8 \%$ contrast & $16 \%$ contrast & $32 \%$ contrast \\
\hline$n=21(24)$ & $5.76(4.00)$ & $5.29(6.5)$ & $1.93 \pm 2.73(1.80)$ & $-5.74 \pm 9.64(-4.15)$ \\
\hline
\end{tabular}

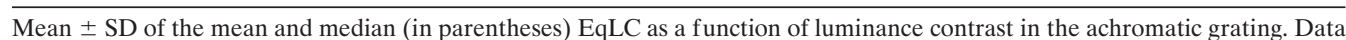
are presented for the two monkeys ( $\mathrm{T}$ and $\mathrm{K}$ ) separately. The leftmost column shows the number of neurons for which EqLC could be determined and (in parentheses) the number of neurons that were investigated using the respective stimulus configuration. EqLC SD is presented for stimulus conditions in which nulling occurred for all cells.

two-way ANOVA was used separately for each achromatic luminance contrast to detect dependencies of EqLC on temporal frequency (factor 1), and spatial frequency (factor 2). For each temporal frequency, the EqLC was found to decrease with increasing luminance contrast in the achromatic grating. No relationship between temporal frequency and EqLC was detected $(p>0.05)$.

Mean EqLC is plotted in Figure $10 B$ as a function of achromatic luminance contrast ( $x$-axis) for neurons tested at different spatial frequencies. As seen for the temporal frequency data, a decrease in EqLC with increasing achromatic luminance contrast was observed for all spatial frequencies. In contrast to temporal frequency data, the ANOVA revealed a significant dependence of EqLC on spatial frequency $(p<0.05)$ at all achromatic luminance contrasts. EqLCs were highest for neurons that preferred $0.7 \mathrm{cyc} /{ }^{\circ}$ and lowest for neurons that preferred $1.4 \mathrm{cyc} /{ }^{\circ}$. Post hoc paired comparisons (Tukey's test) revealed that EqLCs for neurons preferring 0.4 and $0.7 \mathrm{cyc} /{ }^{\circ}$ did not differ from one another, but both were significantly different $(p<0.05)$ from EqLC for neurons that preferred $1.4 \mathrm{cyc} /{ }^{\circ}$. Qualitatively similar results were obtained for neurons recorded with only two achromatic contrasts-i.e., EqLCs were lowest for neurons that preferred 1.4 cyc $/{ }^{\circ}$ - although the effect did not reach significance.

Because EqLC was only measured for the single spatiotemporal frequency determined to be preferred through preliminary testing (see Materials and Methods), the analysis presented here confounds preferred and tested frequency conditions. Because of the preferred-tested confound, it is impossible to determine from these data whether (1) all sampled neurons with higher spatial frequency preferences exhibit less sensitivity to chromatic contrast or (2) all sampled neurons exhibit less sensitivity to chromatic contrast when tested at higher spatial frequencies. Both are 
A

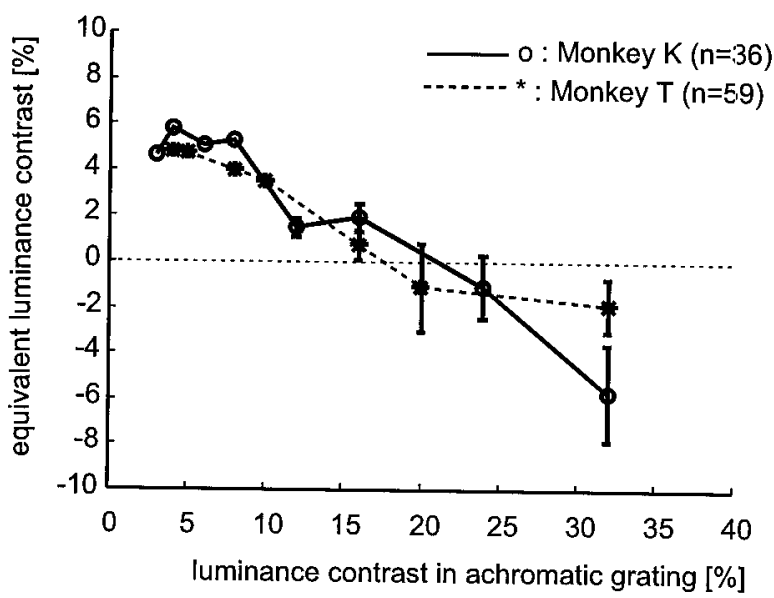

B

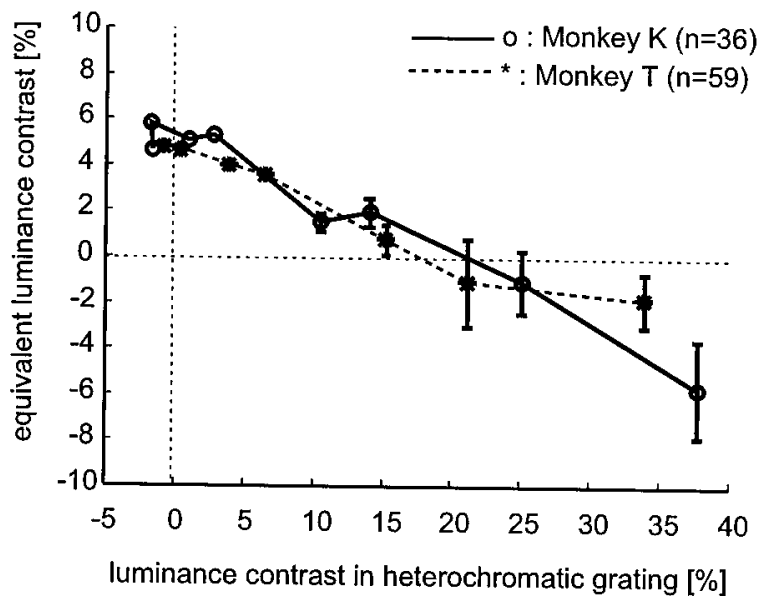

Figure 9. Population EqLC: inverse relationship between average EqLC and actual luminance contrast in the opposed-motion stimulus. $A$, Mean EqLC $\pm \mathrm{SE}$ are plotted as a function of luminance contrast amplitude in the achromatic component of the opposed-motion stimulus. Data are averaged and plotted separately for the two monkeys. Average EqLC declined in an approximately linear manner as luminance contrast was increased. Although significantly positive at $3-5 \%$ contrast (i.e., when the achromatic component was small), average EqLC became effectively negative when luminance contrast exceeded 17-20\%. B, Data from $A$ have been replotted as a function of luminance contrast in the heterochromatic grating. This was achieved by subtracting EqLC from achromatic luminance contrast. Replotting the data in this manner is advantageous, because it allows one to appreciate more intuitively the variable relationship between neuronal sensitivity to the chromatic (as expressed by EqLC, $y$-axis) and luminance ( $x$-axis) components of a moving heterochromatic pattern. Note that the negative luminance contrast values plotted on the $x$-axis do not indicate "red more luminous than green" (as was the case for Figs. 2, 3, 5-7). Rather, negative values occurred simply because the heterochromatic component often dominated the response when the achromatic component was of low contrast (i.e., no null points were exhibited; e.g., see Fig. $5 A$ ). In such cases, EqLC was estimated by a lower boundary, as described in Materials and Methods. Negative heterochromatic luminance contrast values were thus assigned when the procedure yielded EqLCs that were larger than the achromatic test contrast. In both $A$ and $B$, error bars are not plotted for low luminance contrasts $(\leq 10 \%)$, because the heterochromatic grating generally dominated the neuronal response under these conditions, and no null points were observed. In this case EqLC was approximated as the achromatic luminance contrast or the highest EqLC obtained for this neuron. intriguing possibilities with important mechanistic implications and will require further study.

\section{Psychophysical estimates of equivalent luminance contrast}

The observed decline of EqLC as a function of luminance contrast for MT neurons was somewhat unexpected, because Cavanagh and Anstis (1991) reported that psychophysically determined values of EqLC for human subjects were primarily independent of luminance contrast. There are a number of possible explanations for this difference, which include (1) the use of somewhat different stimulus configurations (e.g., spatial and temporal frequency, eccentricity, and size), (2) a species difference (human vs macaque), and (3) the hypothesis that MT accounts for this perceptual phenomenon may be invalid. The first of these, stimulus differences, is easy to address experimentally, and we did so by determining the psychophysical EqLCs of two human subjects (A.T. and a naïve subject M.M.) using stimulus conditions that were as similar as possible (see Materials and Methods) to those used for our studies of single neurons in macaque. Psychophysical results are plotted in Figure 11. For both subjects, EqLC was in the range of $2-3 \%$ for all achromatic luminance contrasts tested.

In marked contrast to our neuronal data, psychophysically determined EqLC did not decline with increasing achromatic luminance contrast. This psychophysical result is qualitatively consistent with previous reports from Cavanagh and Anstis (1991), which leads us to discount explanations for the neuronal and perceptual discrepancy that are based on simple stimulus differences. We will consider other possible explanations for the discrepancy in some detail in Discussion.

\section{DISCUSSION}

The results from these experiments confirm that MT neurons exhibit directionally selective responses to heterochromatic (redgreen) moving patterns. Our results, moreover, calibrate the strength of this chromatic sensitivity by referring it directly to a luminance benchmark. Doing so has enabled us to assess the degree to which chromatic sensitivity depends on the luminance contrast in a moving pattern and has facilitated comparisons between neuronal and perceptual sensitivity.

We will compare our results with those obtained from previous physiological and psychophysical studies. Additionally we will consider the degree to which our results can be accounted for by magnocellular versus parvocellular inputs. Before proceeding, however, we first evaluate potential confounding factors and attempt to discount the possibility that they have contributed to the observed effects.

\section{Potential confounding factors}

Because of chromatic aberration of the lens, colors intended to be isoluminant can be rendered nonisoluminant in the retinal image. This artifactual contrast is minimal for gratings $<1.0 \mathrm{cyc} /{ }^{\circ}$ but potentially troublesome for higher spatial frequencies (Flitcroft, 1989; Cavanagh and Anstis, 1991). It is thus possible that artifactual contrast was present for our $1.4 \mathrm{cyc} /{ }^{\circ}$ gratings, thereby increasing the apparent driving power of the heterochromatic stimulus. Were this true, EqLC should be largest at this frequency. This prediction is in direct opposition to the pattern observed (Fig. 10). Thus, we argue that chromatic aberration was not a significant confound.

There are a number of other ways in which isoluminant stimuli might create luminance contrast signals in the eye. These include 
A

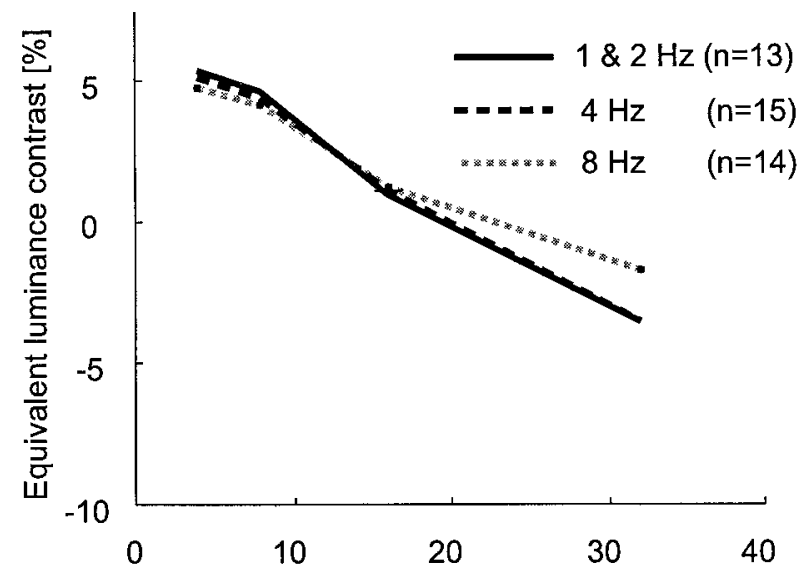

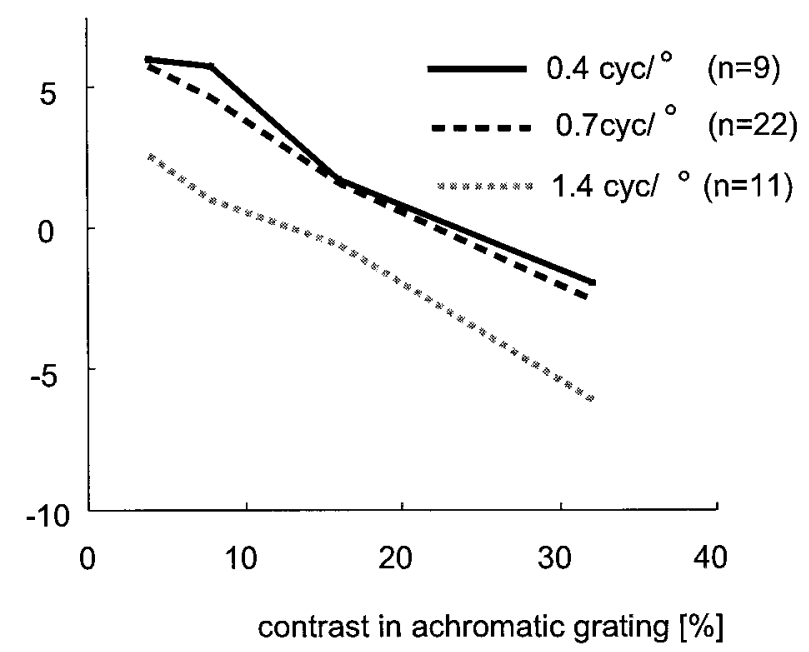

Figure 10. Dependence of EqLC on temporal and spatial frequency (tested with a 2-factor ANOVA: factor one, temporal frequency; factor two, spatial frequency). $A$, Relationship between EqLC and preferred temporal frequency. EqLC is plotted as a function of achromatic luminance contrast in the opposed-motion stimulus, separately for cells of different preferred temporal frequencies. Average EqLC values were similar and decreased with increasing luminance contrast at similar rates for all temporal frequencies tested. Neurons that preferred temporal frequencies of 1 and $2 \mathrm{~Hz}$ were pooled to increase this data set. The EqLC was independent on temporal frequency $(p>0.05)$. B, Relationship between EqLC and preferred spatial frequency. EqLC is plotted as a function of achromatic luminance contrast in the opposed-motion stimulus, separately for cells that preferred each of three different spatial frequencies. Although EqLC values decreased with increasing luminance contrast at similar rates, the average values were significantly smaller for cells that preferred the highest spatial frequency tested $(p<0.05)$. For both $A$ and $B$, note that EqLC was only measured for the single spatiotemporal frequency determined to be preferred through preliminary testing (see Materials and Methods). As a result, it is impossible to determine whether the significant effects observed in $B$ indicate that (1) all sampled neurons with higher spatial frequency preferences exhibit less-positive sensitivity to chromatic contrast, or (2) all sampled neurons exhibit less-positive sensitivity to chromatic contrast when tested at higher spatial frequencies.

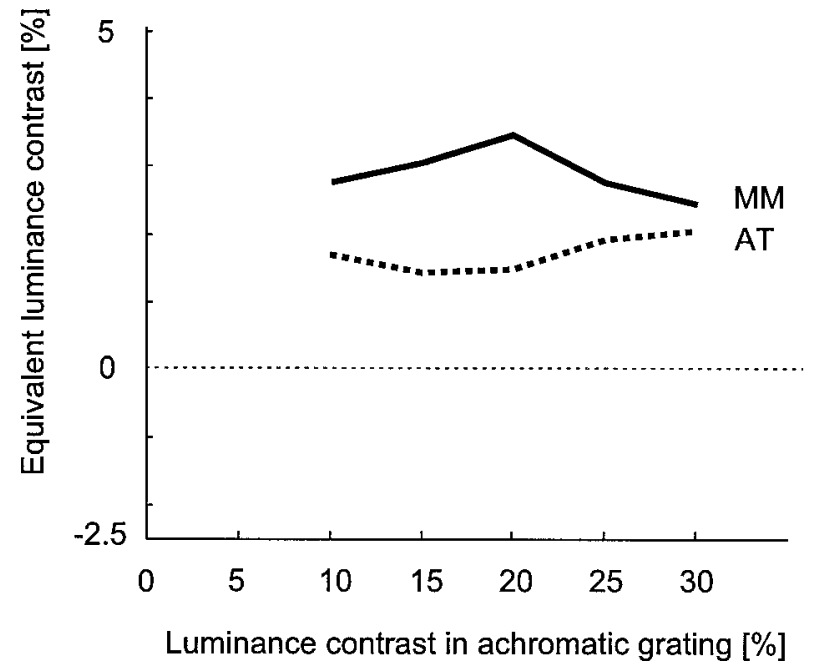

Figure 11. Human psychophysical EqLC as a function of luminance contrast for two human subjects. EqLC was determined using stimuli of $0.7 \mathrm{cyc} /{ }^{\circ}$ spatial and $4 \mathrm{~Hz}$ temporal frequency. Stimulus size was $4.7^{\circ}$ in diameter, and stimuli were centered $3.7^{\circ}$ to the right of center of gaze. EqLC was primarily independent of luminance contrast for both subjects. Minimum EqLC was 1.43; maximum EqLC was $3.5 \%$.

rod intrusion (Mullen, 1991) and inhomogeneities in the degree of macular pigment (Stabell and Stabell, 1980) or ratios of L:M cones (e.g., Nerger and Cicerone, 1992). As we have noted previously (Dobkins and Albright, 1993, 1994), luminance signals rendered by such factors are unlikely to explain motion percep- tion or directionally selective MT responses at red-green isoluminance.

\section{Chromatic input to MT: electrophysiological studies}

Previous experiments have shown that MT neurons are sensitive to the motion of heterochromatic (red-green) patterns (Charles and Logothetis, 1989; Saito et al., 1989; Dobkins and Albright, 1990, 1994; Gegenfurtner et al., 1994; Dobkins et al., 1998; Croner and Albright, 1999). Two studies, in particular, have attempted to quantify the strength of this sensitivity.

Dobkins and Albright $(1994,1998)$ compared neuronal responses elicited by individual achromatic and heterochromatic moving gratings. From these data, they determined the luminance contrast difference between the two stimulus types required to elicit equivalent degrees of directional selectivity, a metric from which EqLC can be inferred. Isoluminant heterochromatic stimuli yielded direction indices, similar to achromatic stimuli of 2-3\% luminance contrast. As luminance contrast was added to the heterochromatic stimuli, sensitivity to its chromatic component declined, becoming nil when luminance contrast exceeded $5 \%$. In this latter respect, the results of Dobkins and Albright (1994, 1998) are qualitatively similar to those reported herein. However, EqLCs from the former study were smaller on average and reached zero at lower luminance contrast. This discrepancy is likely attributable to stimulus differences. In particular, Dobkins and Albright (1994) used stimuli in which motion could only be detected by a mechanism sensitive to the sign of chromatic contrast (red-green gratings were phase-shifted in $90^{\circ}$ increments). Stimuli used in the present study, by contrast, offered both chromatic sign and chromatic borders as cues for motion corre- 
A

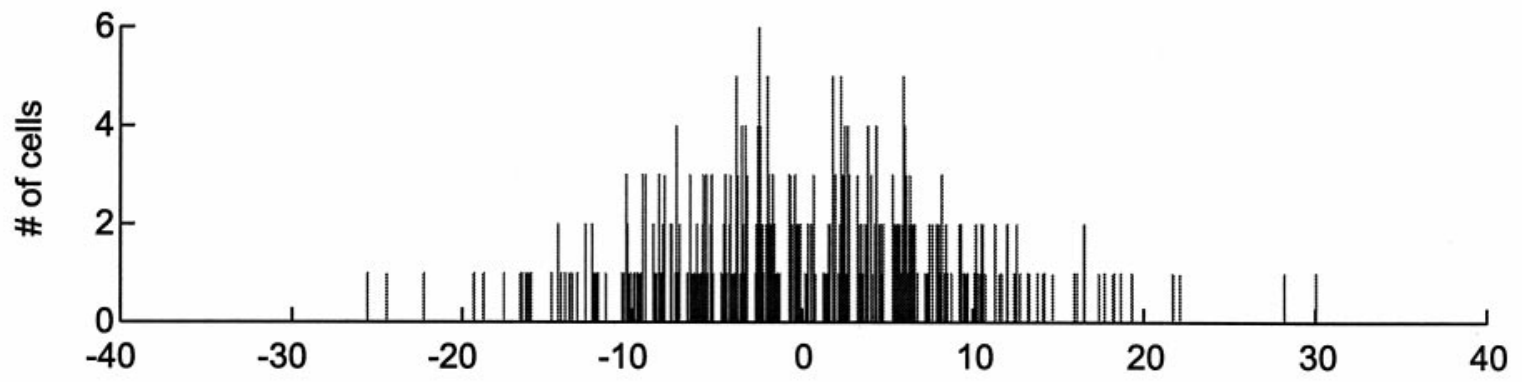

B

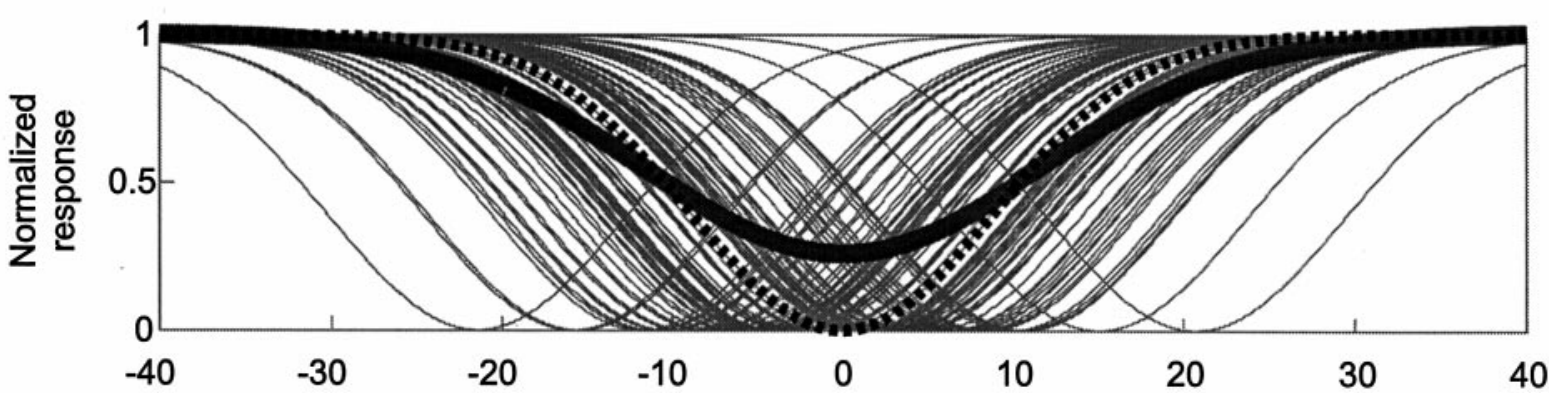

C

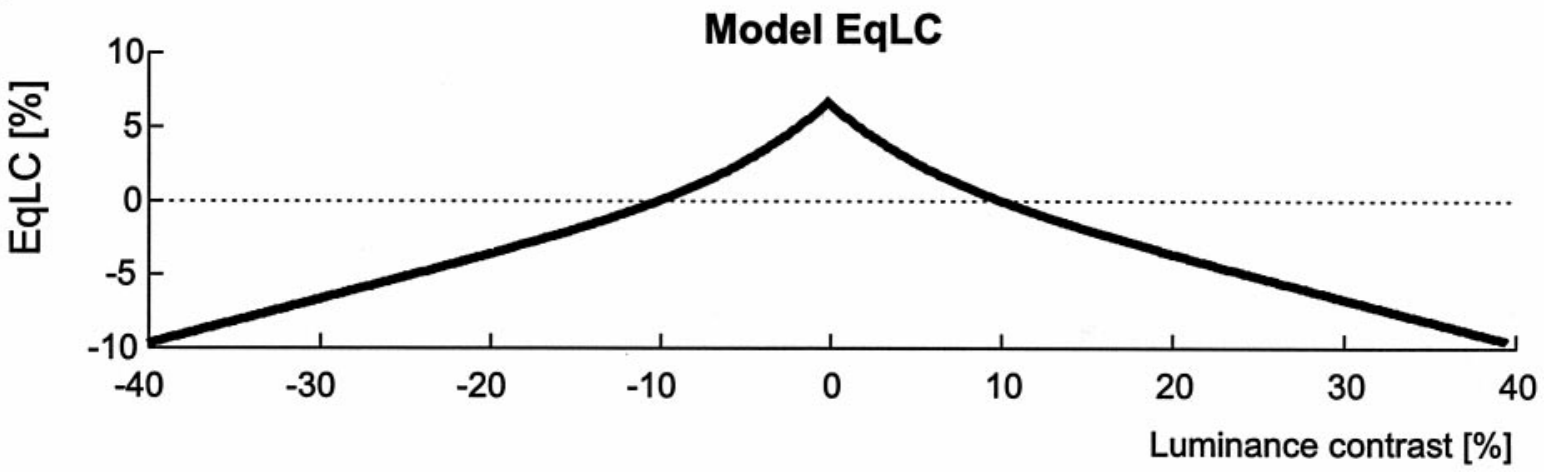

D

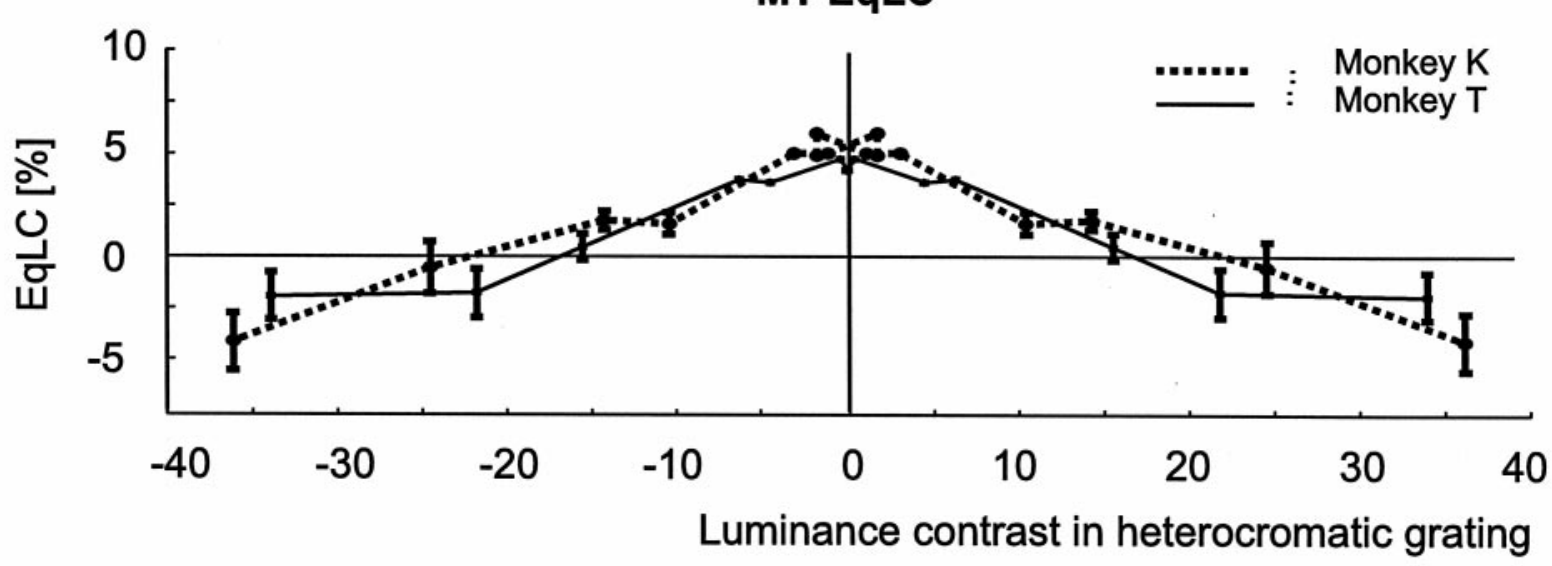

Figure 12. M-cell input model of MT EqLC. M-cell pooling model accounts for the observed relationship between neuronal EqLC and luminance contrast. $A$, Modeled distribution of isoluminant points for M-cells in primate lateral geniculate nucleus (LGN). The units of the $x$-axis correspond to heterochromatic luminance contrast values for a red-green temporally modulated pattern. Isoluminant points are the luminance contrast values for red and green lights that elicit neuronal responses of equivalent magnitude. In general agreement with published data (Logothetis et al., 1990), we performed a simulation assuming that individual M-cells have scattered isoluminant points. This scatter in our model is assumed to be Gaussian, with an SD of 8\% luminance contrast. MT neurons are assumed to receive inputs that reflect pooled activity from these model M-cells. (For this modeling exercise we make no assumptions about the anatomical site of LGN convergence but, rather, simply assume that the signal reaching an (Figure legend continues) 
spondence. The latter is likely to have bolstered the chromatic response and hence EqLC.

In another study, Gegenfurtner et al. (1994) compared motion identification thresholds obtained from a macaque monkey with responses of directionally selective MT neurons, both of which were elicited by moving achromatic and heterochromatic gratings of various luminance contrasts. Based on differences observed, these authors concluded that MT cannot account for the perception of slowly moving chromatic stimuli. This conclusion was founded, in part, on the observation that most MT neurons in the sample did not respond to moving gratings at isoluminance. This latter observation differs from the results of Dobkins and Albright (1994) and those of the present study. The smaller fraction of responsive cells observed by Gegenfurtner et al. (1994) may be a consequence of the lower chromatic contrast present in the stimuli they used.

In sum, the data across studies clearly demonstrate chromatic influences on MT responses. Where discrepancies exist, regarding the strength of chromatic sensitivity, they are most likely attributable to differences in visual stimuli and experimental design. The value of the present approach thus lies in its ability to provide a precise quantitative assessment of chromatic influences and, most importantly, to be applicable to both physiological and psychophysical measurements of EqLC.

\section{Chromatic input to motion perception: psychophysical estimates of EqLC}

To quantify the strength of human perceptual sensitivity to motion of heterochromatic stimuli, Cavanagh and Anstis (1991) developed the opponent motion paradigm on which the present experiments were based. Using this approach, EqLC was measured across a variety of spatiotemporal frequencies (a subset of the range investigated herein). For red-green gratings of low spatiotemporal frequency, perceptual EqLC was $\sim 10 \%$, and this value was primarily independent of luminance contrast, a manifestation, they argued, of a color opponent ( $\mathrm{P}$ pathway) contribution. In stark contrast to these psychophysical results, we have observed neuronal EqLC to be primarily dependent on luminance contrast in a moving heterochromatic grating. To address the possibility that different paradigms and stimuli could account for the discrepancy, we repeated these psychophysical experiments using stimulus conditions that were as close as possible to those used in the present physiological study. Our psychophysical results are in general agreement with those of Cavanagh and Anstis (1991)-i.e., EqLC does not decrease with increasing luminance contrast-and thus are also at variance with the behavior of most MT neurons.

\section{Chromatic input to motion processing: parvocellular or magnocellular contribution?}

Sensitivity to the motion of heterochromatic patterns could stem from signals originating in $\mathrm{M}$ and/or $\mathrm{P}$ pathways. Area MT receives input from both, although the largest fraction of its input arises from the M pathway (Maunsell et al., 1990). To explore whether perceptual EqLC could be accounted for by chromatic responses originating within the $\mathrm{M}$ pathway, Cavanagh and Anstis (1991) tested a model based on the variance of M-cell isoluminant points. Contrary to the invariance of perceptual EqLC, the M-cell model predicted that EqLC should decrease with increasing luminance contrast. On the other hand, a model based on color-opponent P-cell responses predicted EqLC to be independent of luminance contrast. Because the latter behavior was consistent with their psychophysical findings, Cavanagh and Anstis (1991) argued that mainly P-cells contribute to chromatic motion perception. Recent psychophysical evidence appears to support this interpretation (Gegenfurtner and Hawken, 1995; Stromeyer et al., 1995; Cropper and Derrington, 1996).

In contrast to these reports, the results of our previous psychophysical (Dobkins and Albright, 1993) and neurophysiological studies (Dobkins and Albright, 1994, 1998) point to a significant M-cell contribution. To address this issue further, we adopted and modified the M-cell model of Cavanagh and Anstis (1991) to determine whether it could account for the physiological data reported herein. In this model, MT neurons pool activity from magnocellular input neurons, which, as a population, have a Gaussian distribution of isoluminance points (Fig. 12A) and individually possess Gaussian response functions (Fig. 12B). According to the model, phase information (red brighter than green or vice versa) carried by individual magnocellular neurons is discarded; i.e., signals reaching MT are rectified with respect to sign of chromatic contrast.

The heterochromatic response of an individual MT neuron, which pools and normalizes the rectified activity of modeled M-cell inputs, is shown by the stippled line in Figure $12 \mathrm{~B}$. Owing to the diversity of $\mathrm{M}$-cell isoluminance points, the response is nonzero for all heterochromatic luminance contrast values. The response of the MT neuron to an achromatic grating is indicated by the solid black line. EqLC of the modeled neuron is defined as the luminance difference between achromatic and heterochromatic gratings that elicit equivalent neuronal responses. EqLC modeled in this manner is plotted in Figure $12 \mathrm{C}$. To facilitate comparison between the model and actual MT data, we have replotted the latter in Figure $12 \mathrm{D}$. As can be seen, there is close correspondence between EqLC of modeled MT neurons and

\footnotetext{
MT neuron is a sum of the signals arising from many LGN neurons.) A total of 400 such M-cell isoluminant points were randomly drawn from the distribution, of which only every third is plotted (the thick curves in $B$ and EqLC in $C$, however, were calculated from the whole sample). $B$, Modeled M-cell and MT neuron responses as a function of luminance contrast in heterochromatic and achromatic patterns. Modeled response functions for a population of M-cells with isoluminant points that vary according to a distribution as shown in $A$ are indicated by numerous solid light gray curves. The response function of each neuron is assumed to be Gaussian, with a slope of 0.08 , indicating that the neuronal response saturates once luminance contrasts of $\sim 25-30 \%$ are obtained. Modeled response functions for MT neurons that receive inputs that reflect pooled M-cell activity are shown for achromatic (broken curve) and heterochromatic (solid dark gray curve). The achromatic MT response curve drops to zero at zero luminance contrast and is reflected around the $0 \%$ contrast point to facilitate comparison with the heterochromatic response function. Because of variance in the distribution of M-cell isoluminance points shown in $A$, the MT neuron exhibits a nonzero response at all heterochromatic luminance contrast values. $C$, Equivalent luminance contrast of the MT neuron as a function of luminance contrast. The EqLC is obtained by taking the luminance contrast difference in the achromatic and the heterochromatic gratings that give rise to the same neuronal response (as plotted in $B$ ). For low luminance contrasts model EqLC is profound $(\sim 6 \%)$; however, it sharply wanes once luminance contrast is increased. $D$, Average neuronal EqLC as presented in Figure $9 B$ replotted to allow for a comparison with the model. The model behavior is strikingly similar to the MT data; thus the results from this model suggest that magnocellular input to MT is sufficient to account for the results reported herein.
} 
those recorded in the present study. Thus, in accordance with our previous experiments (Dobkins and Albright, 1994), the results from this model suggest that magnocellular input is sufficient to account for the responses of most MT neurons to motion of heterochromatic stimuli.

\section{Is MT the neural basis for chromatic motion processing revealed perceptually?}

The most direct way to assess whether MT is responsible for chromatic motion processing revealed behaviorally would be to compare estimates of EqLC obtained from MT neurons with those obtained from the same monkey tested in a behavioral paradigm (studies that are currently under way in our laboratory). For now, we compare our macaque MT data with psychophysical data obtained from human observers. This comparison is justified because color and motion vision of humans is similar to that of macaques (De Valois et al., 1974; Golomb et al., 1985; Newsome and Paré, 1988).

This comparison reveals marked differences between neuronal responses and psychophysical performance, in that EqLC declines with increasing luminance contrast, whereas perceptual EqLC does not. There are at least four possible explanations for this discrepancy: (1) MT may not be the sole substrate for chromatic motion processing revealed perceptually; (2) only a subset of MT neurons may serve this perceptual phenomenon; (3) chromatic motion processing may be different in monkeys and humans; and (4) chromatic motion processing in MT may be substantially different when monkeys are required to attend to the visual stimulus.

The first of these possibilities, that MT may not be the sole substrate for chromatic motion processing revealed perceptually, was initially suggested by Gegenfurtner et al. (1994) after a comparison of neuronal and psychophysical responses to moving chromatically defined patterns. For slowly moving gratings $(<1$ $\mathrm{Hz}$ ), these investigators found chromatic contrast thresholds for MT neurons to be much higher (by approximately threefold) than those revealed behaviorally. Although the degree to which the conclusion of Gegenfurtner et al. (1994) is a relevant precedent for the present results is unclear, owing to some significant stimulus differences, it nonetheless supports the hypothesis that there may be distinct contributions from other areas of visual cortex to chromatic motion perception. In addition to possessing directionally selective neurons, we should expect prospective areas to receive substantial $\mathrm{P}$ pathway input (the invariance of psychophysical EqLC with increasing luminance contrast is the signature of a color-opponent mechanism; Cavanagh and Anstis, 1991). A potential candidate is area V4, which receives approximately equal M- and P-cell input (Ferrera et al., 1992, 1994a) and contains a respectable number of directionally selective units (Desimone and Ungerleider, 1986; Ferrera et al., 1994b).

The second possible explanation for the neuronal and perceptual difference observed in the present study is that the relatively small number of neurons that exhibited EqLCs that were independent of luminance contrast may constitute a separate population responsible for the perception of heterochromatic motion. It is difficult to evaluate this possibility directly at the present time, but it remains worthy of consideration.

The third and fourth possible reasons for the discrepancy between neuronal and perceptual EqLC are that measurements were made in different primate species and with different behavioral demands. Unlike the case for human psychophysical subjects, monkeys were not required to report direction of stimulus motion. This difference in behavioral requirements presumably leads to a difference in the allocation of attention, which may account for differences in relative sensitivity to chromatic and luminance components (Cavanagh, 1992). Although the present results provide a critical baseline for chromatic motion processing in MT under "fixation-only" conditions, it will obviously be important for future studies to assess neuronal EqLC while simultaneously determining perceptual EqLC from the monkey's own behavioral report.

\section{REFERENCES}

Cavanagh P (1992) Attention-based motion perception. Science 257:1563-1565.

Cavanagh P, Anstis S (1991) The contribution of color to motion in normal and color-deficient observers. Vision Res 31:2109-2148.

Cavanagh P, Tyler CW, Favreau OE (1984) Perceived velocity of moving chromatic gratings. J Opt Soc Am 1:893-899.

Charles ER, Logothetis NK (1989) The responses of middle temporal (MT) neurons to isoluminant stimuli. Invest Ophthalmol Vis Sci 30:427.

Croner LJ, Albright TD (1997) Image segmentation enhances discrimination of motion in visual noise. Vision Res 37:1415-1427.

Croner LJ, Albright TD (1999) Segmentation by color influences responses of motion-sensitive neurons in the cortical middle temporal visual area. J Neurosci 19:3935-3951.

Cropper SJ, Derrington AM (1996) Rapid colour-specific detection of motion in human vision. Nature 379:72-74.

Dacey DM (1996) Circuitry for color coding in the primate retina. Proc Natl Acad Sci USA 93:582-588.

Derrington AM, Badcock DR (1985) The low level motion system has both chromatic and luminance inputs. Vision Res 25:1879-1884.

Derrington AM, Lennie P (1984) Spatial and temporal contrast sensitivities of neurones in lateral geniculate nucleus of macaque. J Physiol (Lond) 357:219-240.

Derrington AM, Krauskopf J, Lennie P (1984) Chromatic mechanisms in lateral geniculate nucleus of macaque. J Physiol (Lond) 357:241-265.

Desimone R, Ungerleider LG (1986) Multiple visual areas in the caudal superior temporal sulcus of the macaque. J Comp Neurol 248:164-189.

De Valois RL, Morgan HC, Polson MC, Mead WR, Hull EM (1974) Psychophysical studies of monkey vision. I. Macaque luminosity and color vision tests. Vision Res 14:53-67.

Dobkins KR, Albright TD (1990) Color facilitates motion in visual area MT. Soc Neurosci Abstr 16:1220.

Dobkins KR, Albright TD (1993) What happens if it changes color when it moves? Psychophysical experiments on the nature of chromatic input to motion detectors. Vision Res 33:1019-1036.

Dobkins KR, Albright TD (1994) What happens if it changes color when it moves? The nature of chromatic input to macaque visual area MT. J Neurosci 14:4854-4870.

Dobkins KR, Albright TD (1998) The influence of chromatic information on visual motion processing in the primate visual system. In: Higher level motion processing-computational, neurobiological, psychophysical perspectives (Watanabe T, ed), pp 53-98. Boston: MIT.

Ferrera VP, Nealey TA, Maunsell JHR (1992) Mixed parvocellular and magnocellular geniculate signals in visual area V4. Nature 358:756-758.

Ferrera VP, Nealey TA, Maunsell JHR (1994a) Responses in macaque visual area V4 following inactivation of the parvocellular and magnocellular LGN pathways. J Neurosci 14:2080-2088.

Ferrera VP, Rudolph KK, Maunsell JHR (1994b) Responses of neurons in the parietal and temporal visual pathways during a motion task. J Neurosci 14:6171-6186.

Flitcroft DI (1989) The interactions between chromatic aberration, defocus and stimulus chromaticity: implications for visual physiology and colorimetry. Vision Res 29:349-360.

Gegenfurtner KR, Hawken MJ (1995) Temporal and chromatic properties of motion mechanisms. Vision Res 35:1547-1563.

Gegenfurtner KR, Kiper DC, Beusmans JMH, Caradini M, Zaidi Q, Movshon JA (1994) Chromatic properties of neurons in macaque MT. Vis Neurosci 11:455-466.

Golomb B, Andersen RA, Nakayama K, MacLeod DI, Wong A (1985) Visual thresholds for shearing motion in monkey and man. Vision Res 25:813-820. 
Judge SJ, Richmond BJ, Chu FC (1980) Implantation of magnetic search coils for measurement of eye position: an improved method. Vision Res 20:535-538.

Kaiser PK, Lee BB, Martin PR, Valberg A (1990) The physiological basis of the minimally distinct border demonstrated in the ganglion cells of the macaque retina. J Physiol (Lond) 422:153-183.

Lee BB, Martin PR, Valberg A (1988) The physiological basis of heterochromatic flicker photometry demonstrated in the ganglion cells of the macaque retina. J Physiol (Lond) 404:323-347.

Lee BB, Martin PR, Valberg A (1989a) Nonlinear summation of M- and L-cone inputs to phasic retinal ganglion cells of the macaque. J Neurosci 9:1433-1442.

Lee BB, Martin PR, Valberg A (1989b) Sensitivity of macaque retinal ganglion cells to chromatic and luminance flicker. J Physiol (Lond) 414:223-243.

Lindsey DT, Teller DY (1990) Motion at isoluminance: discrimination/ detection ratios for moving isoluminant gratings. Vision Res 30:1751-1761.

Logothetis NK, Schiller PH, Charles ER, Hurlbert AC (1990) Perceptual deficits and the activity of the color-opponent and broad-band pathways at isoluminance. Science 247:214-217.

Maunsell JHR, Nealey TA, DePriest DD (1990) Magnocellular and parvocellular contributions to responses in the middle temporal area (MT) of the macaque monkey. J Neurosci 10:3323-3334.

Merigan WH, Maunsell JHR (1993) How parallel are the primate visual pathways. Ann Rev Neurosci 16:369-402.

Mullen KT (1991) Colour vision as a post-receptoral specialization of the central visual field. Vision Res 31:119-130.

Mullen KT, Baker CLJR (1985) A motion aftereffect from an isoluminant stimulus. Vision Res 25:685-688.

Murphy BJ, Kowler E, Steinman RM (1975) Slow oculomotor control in the presence of moving backgrounds. Vision Res 15:1263-1268.

Nealey TA, Maunsell JHR (1994) Magnocellular and parvocellular contributions to the responses of neurons in macaque striate cortex. J Neurosci 14:2069-2079.

Nerger JL, Cicerone CM (1992) The ratio of L cones to M cones in the human parafoveal retina. Vision Res 32:879-888.
Newsome WT, Paré EB (1988) A selective impairment of motion perception following lesions of the middle temporal visual area (MT). J Neurosci 8:2201-2211.

Press WH, Flanner BP, Teukolsky SA, Vetterling WT (1992) Numerical recipes in C. Cambridge: Cambridge UP.

Robinson DA (1963) A method of measuring eye movement using a scleral search coil in a magnetic field. IEEE Trans Biomed Eng 10:137-145.

Saito H, Tanaka K, Isono H, Yasuda M, Mikami A (1989) Directionally selective responses of cells in the middle temporal area (MT) of the macaque monkey to the movement of equiluminous opponent color stimulus. Exp Brain Res 75:1-14.

Sawatari A, Callaway EM (1996) Convergence of magno- and parvocellular pathways in layer 4B of macaque primary visual cortex. Nature 380:442-446.

Schiller PH, Colby CL (1983) The responses of single cells in the lateral geniculate nucleus of the rhesus monkey to color and luminance contrast. Vision Res 23:1631-1641.

Schiller PH, Malpelli JG (1978) Functional specificity of lateral geniculate nucleus laminae of the rhesus monkey. J Neurophysiol 41:788-797.

Stabell U, Stabell B (1980) Variation in density of macular pigmentation and in short-wave cone sensitivity with eccentricity. J Opt Soc Am 70:706-711.

Stockman A, MacLeod DI, Johnson NE (1993) Spectral sensitivities of the human cones. J Opt Soc Am 10:2491-2521.

Stromeyer III CF, Kronauer RE, Ryu A, Chaparro A, Eskew RTJ (1995) Contributions of human long-wave and middle-wave cones to motion detection. J Physiol (Lond) 485:221-243.

Valberg A, Lee BB, Kaiser PK, Kremers J (1992) Responses of macaque ganglion cells to movement of chromatic borders. J Physiol (Lond) 458:579-602.

Watson AB, Nielson KRK, Poirson A, Fitzhugh A, Bilson A, Nguyen A, Ahumada AJ (1986) Use of raster framebuffer in vision research. Behav Res Methods Instr Comp 18:587-594.

Yoshioka T, Levitt JB, Lund JS (1994) Independence and merger of thalamocortical channels within macaque monkey primary visual cortex: anatomy of interlaminar projections. Vis Neurosci 11:467-489. 